\title{
Novel genetic loci underlying human intracranial volume identified through genome-wide association
}

Intracranial volume reflects the maximally attained brain size during development, and remains stable with loss of tissue in late life. It is highly heritable, but the underlying genes remain largely undetermined. In a genome-wide association study of 32,438 adults, we discovered five previously unknown loci for intracranial volume and confirmed two known signals. Four of the loci were also associated with adult human stature, but these remained associated with intracranial volume after adjusting for height. We found a high genetic correlation with child head circumference ( $\rho_{\text {genetic }}=0.748$ ), which indicates a similar genetic background and allowed us to identify four additional loci through meta-analysis $\left(N_{\text {combined }}=37,345\right)$. Variants for intracranial volume were also related to childhood and adult cognitive function, and Parkinson's disease, and were enriched near genes involved in growth pathways, including PI3K-AKT signaling. These findings identify the biological underpinnings of intracranial volume and their link to physiological and pathological traits.

The intricate genetic control of the human brain, complemented by environmental factors, leads to the observed variations in brain size in human populations ${ }^{1}$. Intracranial volume is closely related to brain volume in early life as the brain grows ${ }^{2,3}$. However, it becomes stable after the brain has fully developed and remains unaffected by later age-related changes such as brain atrophy ${ }^{4,5}$, thereby representing the maximal attained brain size. Discovering genetic variants that influence intracranial volume can contribute to our understanding of brain development and related diseases, but prior studies have only identified two influential genetic loci ${ }^{6-9}$.

We carried out genome-wide association studies (GWAS) in populations from the Cohorts for Heart and Aging Research in Genomic Epidemiology (CHARGE) ${ }^{10}$ and Enhancing NeuroImaging Genetics through Meta-Analysis (ENIGMA) ${ }^{11}$ consortia on intracranial volume, as measured by magnetic resonance imaging. Genotypes were imputed to the 1000 Genomes reference panel (phase 1, version 3). Meta-analysis revealed five previously unknown loci associated with intracranial volume. We also discovered genome-wide overlap between intracranial volume and other key traits, including height, cognitive ability and Parkinson's disease. Furthermore, we found relatively enriched patterns of association for certain functional categories of variants and near genes that were involved in specific pathways.

\section{RESULTS}

GWAS

Detailed information on the population characteristics, image acquisition and processing, and genetic quality control can be found in the Online Methods and Supplementary Tables 1-3.

The discovery meta-analysis $(N=26,577)$ yielded seven genome-wide significant $\left(P<5 \times 10^{-8}\right)$ loci, five of which were previously unknown (Figs. 1 and 2, and Table 1). The quantile-quantile plot showed inflation ( $\lambda=1.092$; Supplementary Fig. 1), which we determined to be mainly a result of polygenicity rather than cryptic relatedness or population stratification using linkage disequilibrium score regres$\operatorname{sion}^{12}$. Next we analyzed European samples $(N=2,362$; not included in the discovery sample) and generalization samples with African $(N=938)$, Asian $(N=955)$ and Hispanic $(N=1,605)$ ancestries (Table 1). All variants in the additional European samples had the same direction of effect (sign test, $P=0.0078$ ), and three variants replicated, at nominal significance. Although sample sizes were generally small for the non-Europeans, here too, the direction of effect was generally concordant with the discovery ( $\operatorname{sign}$ test, $P=0.039$ ). We detected five nominally significant associations across all three ethnicities.

Next we mapped the association to new variants for two previously identified loci at chromosome $17 \mathrm{q} 21\left(\mathrm{rs} 199525 ; P=3.8 \times 10^{-21}\right)$ and $6 \mathrm{q} 22$ $\left(\text { rs11759026; } P=2.2 \times 10^{-20}\right)^{6,7}$. The five loci were located on chr $6 \mathrm{q} 21$

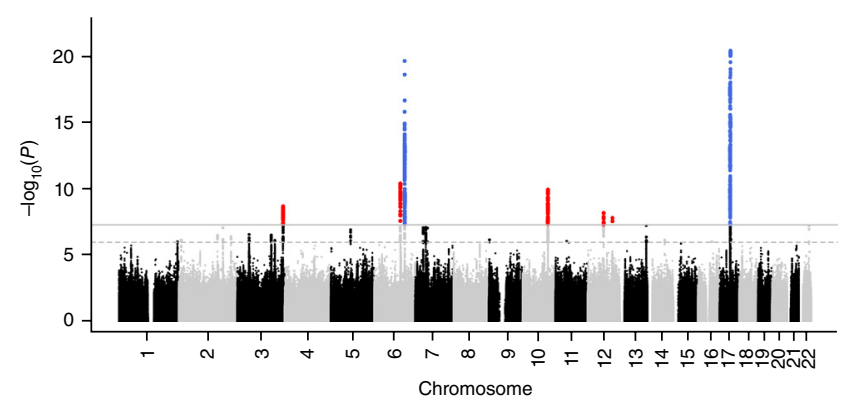

Figure 1 Common genetic variants associated with intracranial volume. Manhattan plot in which every point represents a single genetic variant plotted according to its genomic position ( $x$ axis) and its $-\log _{10}(P)$ for association with intracranial volume ( $y$ axis). Variants in blue were genomewide significant in a previously known locus, whereas red variants reached genome-wide significant for the first time in that locus. The dashed horizontal line represents a significance threshold of $P<10^{-6}$ and the solid horizontal line represents genome-wide significance of $P<5 \times 10^{-8}$. Variants surpassing these thresholds are indicated by larger points. 

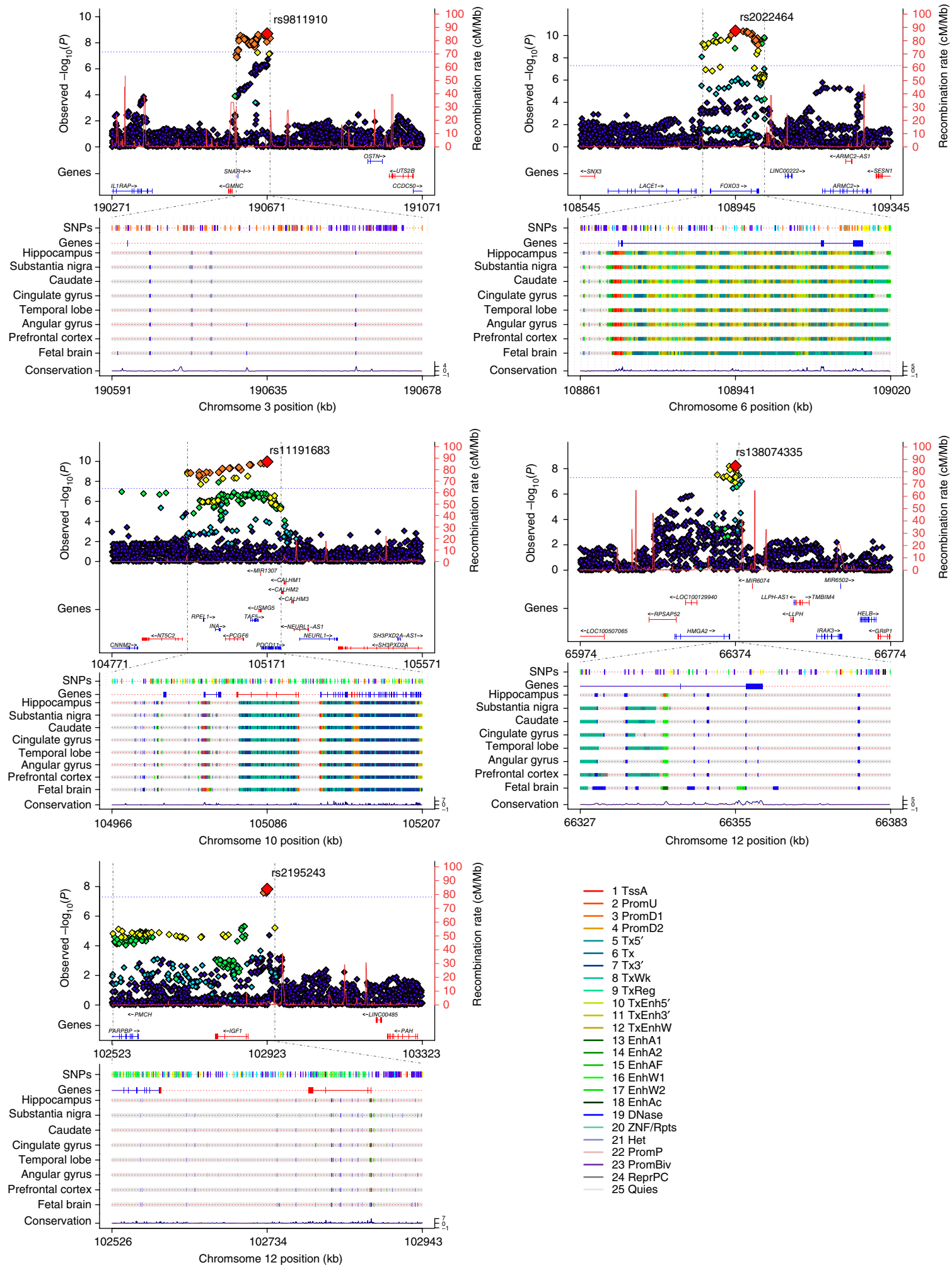

Figure 2 Regional association and functional annotation of previously unknown genome-wide significant loci. Regional association plots for the five genome-wide significant loci of intracranial volume with gene models below (GENCODE version 19). Annotation tracks below from the Roadmap Epigenomics Consortium ${ }^{46}$ highlight the genomic region that likely harbors the causal variant(s) $\left(r^{2}>0.8\right.$ from the top SNP). See Online Methods for detailed track information. Plots were generated using the LocusTrack software (http://gump.qimr.edu.au/general/gabrieC/LocusTrack/).

Color key: 1) Active transcription start site (TSS), 2) Promoter Upstream TSS, 3) Promoter Downstream TSS 1, 4) Promoter Downstream TSS 2 ,

5) Transcribed - 5' preferential, 6) Strong transcription Transcribed - 3' preferential, 7) Weak transcription, 8) Transcribed Regulatory (Prom/Enh), 9) Transcribed 5' preferential and Enh, 10) Transcribed 3' preferential and Enh, 11) Transcribed and Weak Enhancer, 12) Active Enhancer 1, 13) Active Enhancer 2, 14) Active Enhancer Flank, 15) Weak Enhancer 1, 16) Weak Enhancer 2, 17) Primary H3K27ac possible Enhancer, 18) Primary DNase, 19) ZNF genes and repeats, 20) Heterochromatin, 21) Poised Promoter, 22) Bivalent Promoter, 23) Repressed Polycomb, 24) Quiescent/Low. 
Table 1 Association of genome-wide significant loci for intracranial volume in European, African, Asian and Hispanic populations

\begin{tabular}{|c|c|c|c|c|c|c|c|c|c|c|c|c|c|c|c|}
\hline \multirow[b]{2}{*}{ Genetic variant } & \multirow[b]{2}{*}{ Locus } & \multirow[b]{2}{*}{ Position } & \multirow[b]{2}{*}{ Al } & \multirow[b]{2}{*}{ A2 } & \multirow[b]{2}{*}{ Freq } & \multicolumn{2}{|c|}{$\begin{array}{c}\text { European } \\
\text { discovery } \\
(N=26,577)\end{array}$} & \multicolumn{2}{|c|}{$\begin{array}{l}\text { European } \\
\text { replication } \\
(N=2,363)\end{array}$} & \multicolumn{2}{|c|}{$\begin{array}{c}\text { African } \\
\text { generalization } \\
(N=938)\end{array}$} & \multicolumn{2}{|c|}{$\begin{array}{c}\text { Asian } \\
\text { generalization } \\
(N=955)\end{array}$} & \multicolumn{2}{|c|}{$\begin{array}{c}\text { Hispanic } \\
\text { generalization } \\
(N=1,605)\end{array}$} \\
\hline & & & & & & $\beta$ & $P$ & $\beta$ & $P$ & $\beta$ & $P$ & $\beta$ & $P$ & $\beta$ & $P$ \\
\hline 11759026 & $6 q 22$ & 126792095 & A & G & 0.76 & -0.095 & & -0.0 & & & & -0.071 & 0.123 & & 0.209 \\
\hline 2022464 & $6 q 21$ & 108945370 & A & C & 0.30 & -0.063 & $3.7 \times 10^{-11}$ & -0.090 & $4.7 \times 10^{-3}$ & -0.060 & 0.233 & -0.105 & 0.035 & -0.088 & 0.013 \\
\hline rs11191683 & 10q24 & 105170649 & T & G & 0.33 & 0.059 & $1.1 \times 10^{-10}$ & 0.040 & 0.174 & 0.187 & 0.021 & 0.085 & 0.075 & -0.005 & 0.911 \\
\hline
\end{tabular}

A1, effect allele; $A 2$, reference allele; Freq, frequency of the effect allele; $N$, sample size.

(rs2022464; $\left.P=3.7 \times 10^{-11}\right), \operatorname{chr} 10 \mathrm{q} 24\left(\mathrm{rs} 11191683 ; P=1.1 \times 10^{-10}\right)$, chr 3q28 (rs9811910; $\left.P=2.0 \times 10^{-9}\right)$, chr 12q14 (rs138074335/ rs7312464; $\left.P=6.2 \times 10^{-9}\right)$ and $\operatorname{chr} 12 \mathrm{q} 23\left(\mathrm{rs} 2195243 ; P=1.5 \times 10^{-8}\right)$. Functional annotation of the variants and those in LD $\left(r^{2}>0.8\right)$ can be found in Supplementary Table 4.

\section{Height-adjusted analyses}

Four of the seven loci for intracranial volume have previously been discovered for height (17q21, 6q22, 6q21 and 12q14), prompting us to investigate genome-wide overlap between the two traits. Given that height and intracranial volume are correlated (weighted average Pearson's $r=0.556$; Supplementary Table 5), and the possibility that this could drive association signals, we performed a GWAS of intracranial volume adjusted for height in the studies that had measured height $(N=21,875)$. Findings were compared to the corresponding subset of studies without adjustment $(N=22,378)$. Using LD score regression (Online Methods), we found that there was considerable genetic correlation between intracranial volume and height $\left(\rho_{\text {genetic }}\right.$ $\left.=0.241, P=2.4 \times 10^{-10}\right)$, which disappeared after adjusting for height ( $\rho_{\text {genetic }}=0.049, P=0.21$ ) (Table 2$)$. The associations of the seven intracranial volume loci, however, remained significant after adjusting for height (Supplementary Table 6). To investigate whether more height loci were associated with intracranial volume independently of height, we analyzed all 697 genome-wide significant height variants $^{13}$. An additional 73 variants (10.7\%; 14 variants not available) showed nominally significant associations with intracranial volume, but were not attenuated after adjustment for height, although none survived Bonferroni correction (Supplementary Table 7). For some variants, the direction of effect was discordant, that is, positive for height and negative for intracranial volume. Furthermore, a polygenic score of the 697 variants predicted intracranial volume, and this was also the case after adjustment for height in a subset of the studies (Supplementary Table 8).

\section{Genetic correlation}

In addition to height, we examined the genome-wide genetic overlap between intracranial volume and other anthropometric traits, cognitive function, and neurodegenerative diseases (Table 2). We found a strong genetic correlation with child head circumference $\left(\rho_{\text {genetic }}\right.$ $=0.748$ ), which validates intracranial volume as a measure of brain growth during early development. Given that this high correlation indicates that the genetic determinants of intracranial volume and child head circumference are largely shared, we aimed to leverage this information by performing a meta-analysis of both traits. The meta-analysis (combined $N=37,345$ ) led to the identification of four previously unknown loci (Fig. 3 and Supplementary Table 9).

Table 2 Genetic correlation between intracranial volume and other anthropometric traits, cognitive function, and neurodegenerative diseases

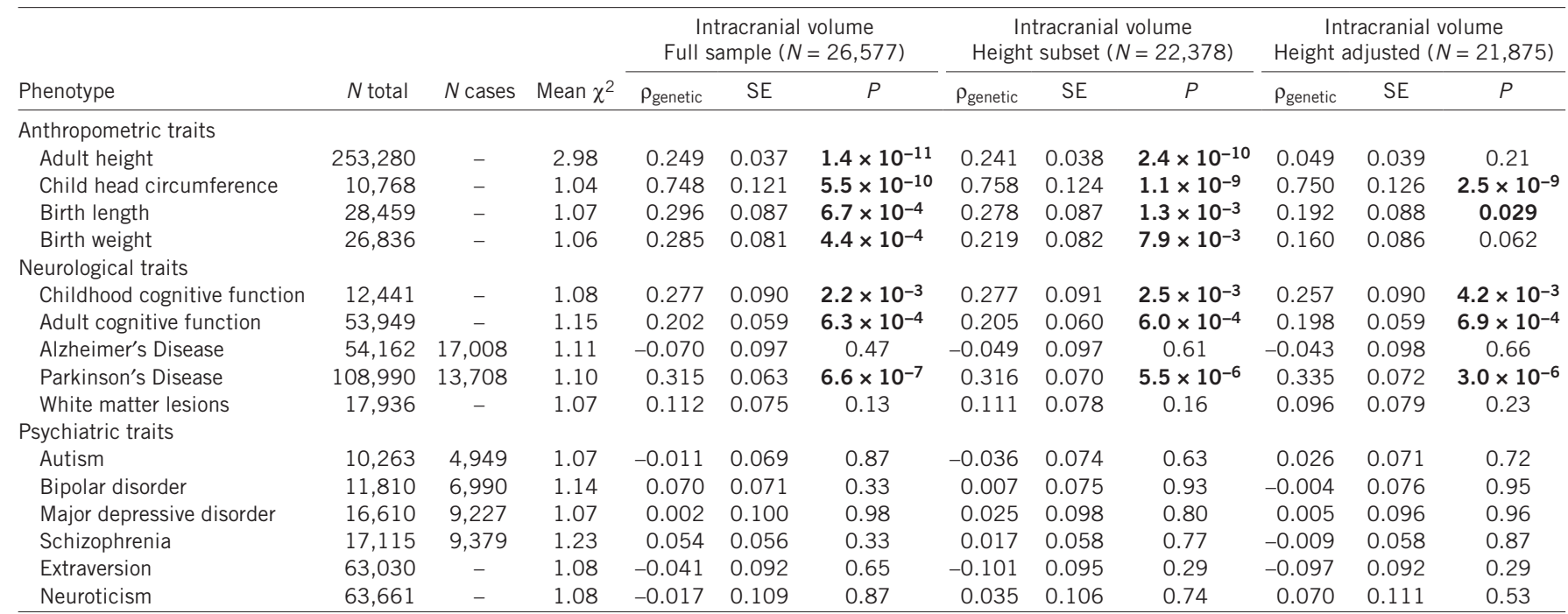

Genetic correlation between various phenotypes and intracranial volume in the complete discovery sample (full sample), adjusted for height in the studies that had measured height (height adjusted), and the corresponding subset of studies without adjustment (height subset). Significant $P$ values are shown in bold. SE, standard error. 


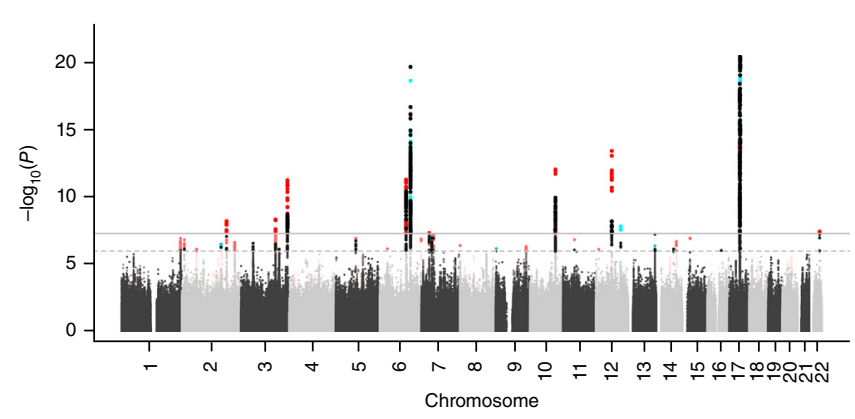

Figure 3 Meta-analysis of intracranial volume and child head circumference. A 'twin' Manhattan plot shows every variant twice: once for the discovery analysis and once for the combined discovery plus replication analysis. The least significant association of the variant-pair is plotted in gray (alternating light and dark between chromosomes). The most significant association of the variant-pair is plotted in red if is from the combined analysis (that is, the association became more significant after meta-analyzing with the child head circumference GWAS) and in turquoise if it is from the discovery analysis (that is, the association became less significant after meta-analyzing with the child head circumference GWAS). The dashed horizontal line represents a significance threshold of $P<10^{-6}$ and the solid horizontal line represents genome-wide significance of $P<5 \times 10^{-8}$. Variants surpassing these thresholds are indicated by larger and brighter points.

Weaker correlations were found with birth length and weight $\left(\rho_{\text {genetic }}<0.3\right.$ ), which attenuated after adjusting for height. In addition, intracranial volume was genetically correlated with cognitive function in childhood $\left(\rho_{\text {genetic }}=0.277, P=2.2 \times 10^{-3}\right)$, as well as general cognitive function in middle-aged and older adults $\left(\rho_{\text {genetic }}\right.$ $\left.=0.202, P=6.3 \times 10^{-4}\right)$. Furthermore, we found a positive genetic correlation with Parkinson's disease $\left(\rho_{\text {genetic }}=0.315, P=6.6 \times 10^{-7}\right)$, but there was no significant genetic overlap with Alzheimer's disease, white matter lesions and psychiatric traits.

\section{Enrichment analyses}

Next, we assessed whether particular subsets of genetic variants were enriched for association with intracranial volume using partitioned heritability and pathway analyses (Online Methods). Overall, we found that common variants genotyped from across the whole genome explained $25.42 \%$ (s.e.m., $2.73 \%$ ) of the variation in intracranial volume. Partitioning heritability by chromosome revealed that chromosome 22 contributed twofold more to variation in intracranial volume than would be expected by its size (Fig. 4a), which was not seen for any of the other complex traits from the genetic correlation analysis (Supplementary Fig. 2). Partitioning by functional elements showed an enrichment for introns and several histone codes that are found in actively transcribed promoters (Fig. $4 \mathbf{b}$ ). The enrichment for intronic variants was specific to intracranial volume, whereas the other functional classes were also enriched in other complex traits (Supplementary Fig. 3). We also found that loci associated with intracranial volume cluster around genes involved in specific pathways, with 94 pathways being significantly enriched (Fig. 4c; full list in Supplementary Table 10). These pathways included all of the cell cycle components-the M, G1, S and G2 phases-and various growth factor signaling pathways, including PI3K-AKT.

\section{Head growth trajectories}

Although intracranial volume reflects brain development until maturation, and we identified influences of many growth-related processes contributing to its variation, all of the loci were still discovered via cross-sectional associations in adults. Thus, we tested whether a polygenic score of the seven loci could predict head growth in a longitudinal cohort of 2,824 children of European ancestry followed prenatally until 6 years of age (Online Methods). We found that a higher polygenic score, representing a genetically larger intracranial volume in adults, was also associated with a larger child head circumference $(\beta=0.031$ per s.d., $P=0.010)$. Furthermore, the effect of the polygenic score was age dependent and more prominent in older children $\left(\beta=0.0080\right.$ per s.d. polygenic score per year age, $P_{\text {interaction }}=$ $0.0091)$. When investigating the individual loci separately, we found significant associations between both $17 \mathrm{q} 21$ and $12 \mathrm{q} 14$ and child head circumference, but they influenced the trajectories of head growth differently (Fig. 5). For 17q21, the negative effect of the G allele on head circumference became apparent postnatally and increased toward 6 years of age, whereas the 12q14 locus exerted an effect from early pregnancy to 1 year of age, but was less prominent later in life.

\section{DISCUSSION}

Genes contributing to variation in the size of the human brain remain challenging to discover. In a worldwide project of unprecedented scale, we performed the largest-ever meta-analysis of GWAS of intracranial volume. We discovered five previously unknown genetic loci associated with intracranial volume, and replicated two known signals. The discovery sample included Europeans only, but the direction of effect was similar in other ethnicities. The genes in these loci provide intriguing links between maximal brain size and various processes, including neural stem cell proliferation (FOXO3), neurodegeneration $(M A P T)$, bone mineralization (CENPW), growth signaling (IGF1, HMGA2), DNA replication (GMNC) and rRNA maturation (PDCD). On a genome-wide scale, we discovered evidence of genetic correlation between intracranial volume and other key traits such as height and cognitive function, as well as with Parkinson's disease, indicating that the genes underlying brain development have far-reaching effects that extend well beyond the initial years of life.

The $17 \mathrm{q} 21$ locus tags a $1-\mathrm{Mb}$ inversion that is under positive selection in Caucasians ${ }^{14}$. It contains multiple genes, including MAPT and KANSL1. The MAPT gene has been consistently implicated in various neurodegenerative disorders, including Parkinson's disease, Alzheimer's disease and frontotemporal dementia ${ }^{15,16}$, and microduplications have been reported to cause microcephaly ${ }^{17}$. KANSL1 causes the reciprocal 17q21.31 microdeletion syndrome: a multisystem disorder characterized by intellectual disability, hypotonia and distinctive facial features ${ }^{18}$. The signal at $6 \mathrm{q} 22$ is intergenic to CENPW and RSPO3, but now lies $172 \mathrm{~kb}$ closer to CENPW. Notably, multiple variants at this locus independently influence bone mineral density ${ }^{19,20}$, and our signal particularly overlapped with the variant showing high specificity for the skull ${ }^{20}$.

The significant variants at chr 6q21 span FOXO3, a gene associated with longevity ${ }^{21}$, height ${ }^{13}$ and serum IGF1 levels ${ }^{22}$. FOXO3 regulates the proliferation of neural stem cells, and knockout mice have larger brains, resulting from increased proliferation immediately after birth ${ }^{23}$, followed by a decrease in adult neural stem cell renewal ${ }^{23,24}$. The rs3800229 variant in strong LD with our top variant $\left(r^{2}=0.84\right)$ contains chromatin promoter marks in the fetal brain (Supplementary Table 4), and regulates serum IGF1 levels in infants ${ }^{25}$. This provides a link to the genome-wide significant locus on chr12q23 near IGF1, pointing to a potential mechanism by which these loci may affect brain growth. Chr12q23 lies $20 \mathrm{Mb}$ from one of two loci previously detected for head circumference in children ${ }^{26}$, but that region was not associated with intracranial volume in our study (rs7980687, $P=0.06$ ). The other reported child head circumference locus, however, corresponded to our chr12q14 signal, with the top 
ARTICLES

a

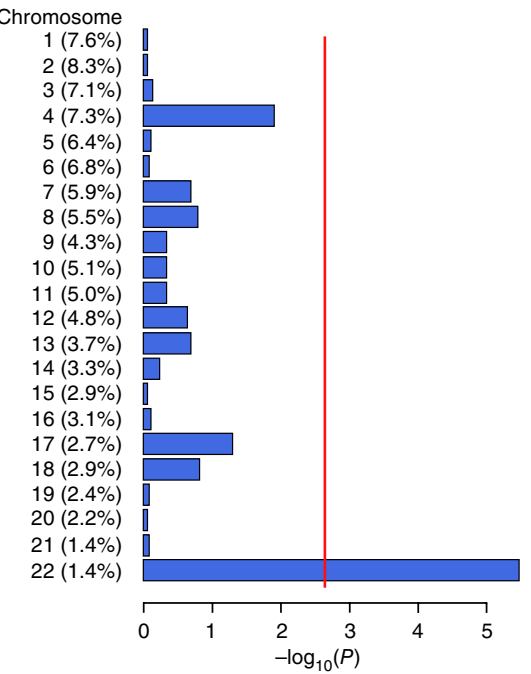

Enrichment
$1.0(0.23)$

$1.0(0.31)$

$0.9(0.35)$

$0.5(0.19)$

$1.0(0.19)$

$0.9(0.41)$

$1.3(0.26)$

$0.5(0.34)$

$1.4(0.52)$

$0.8(0.30)$

$1.3(0.47)$

$1.5(0.42)$

$0.5(0.42)$

$0.8(0.31)$

$0.9(0.55)$

$0.9(0.40)$

$2.5(0.75)$

$0.3(0.51)$

$1.1(0.35)$

$0.9(1.05)$

$0.8(0.77)$

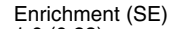

$2.4(0.36)$

$P$ value
0.89
0.89
0.73
0.013
0.8
0.85
0.21
0.16
0.46
0.48
0.47
0.23
0.2
0.59
0.88
0.8
0.05
0.15
0.83
0.92
0.85
$3.4 \times 10^{-6}$

b

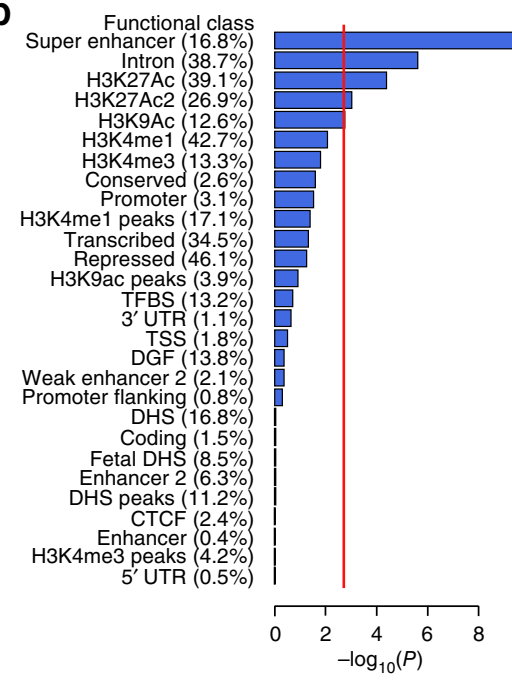

Enrichment (SE) $\begin{array}{ll}\text { Enrichment (SE) } \quad P \text { value } \\ 1.1(0.34) & 4.4 \times 10^{-10}\end{array}$ $\begin{array}{ll}1.8(0.19) & 2.5 \times 10^{-6} \\ 1.9(0.21) & 4.1 \times 10^{-5}\end{array}$ $1.9(0.21) \quad 4.1 \times 10^{-5}$ $2.9(0.56) \quad 9.9 \times 10^{-4}$ $\begin{array}{ll}4.7(1.18) & 1.7 \times 10^{-3} \\ 2.2(0.45) & 7.8 \times 10^{-3}\end{array}$ $2.2(0.45)$
$3.5(1.02)$ $9.7(3.87)$ $6.1(2.45)$
$4.3(1.61)$ $4.3(1.61)$
$2.0(0.52)$ $0.3(0.37)$ $7.5(4.11)$
$3.0(1.57)$ $3.0(1.57)$
$12.0(8.75)$ $6.2(4.90)$ $2.6(1.85)$ $4.5(4.27)$ $6.8(7.57)$ $1.2(1.47)$ $1.5(5.08)$
$1.2(2.53)$ $1.2(2.31)$
$1.2(2.15)$

C

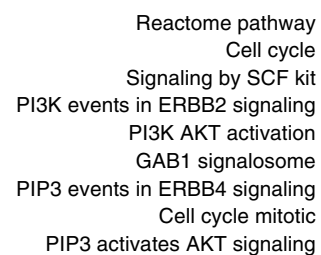

NGF signaling via TRKA Downstream signaling events of $B$ cell receptor BCR Signaling by ERBB2 Signaling by ERBB4 Downstream signal transduction PI3K cascade Signaling by FGFR in disease Signaling by FGFR Downstream signaling of activated FGFR Signaling by PDGF

DNA replication Signaling by NGF

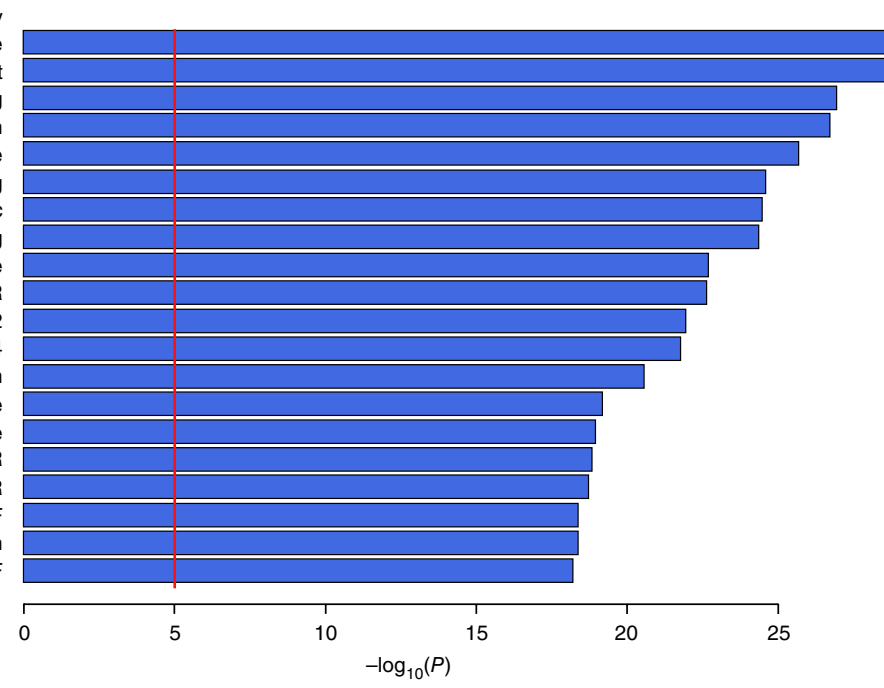

\section{-}

$\begin{array}{rl}N_{\text {Genes }} & P \text { value } \\ 421 & 1.7 \times 10^{-29} \\ 78 & 2.1 \times 10^{-29} \\ 44 & 1.2 \times 10^{-27} \\ 38 & 2.0 \times 10^{-27} \\ 38 & 2.0 \times 10^{-26} \\ 38 & 2.4 \times 10^{-25} \\ 325 & 3.4 \times 10^{-25} \\ 29 & 4.6 \times 10^{-25} \\ 137 & 2.0 \times 10^{-23} \\ 97 & 2.5 \times 10^{-23} \\ 101 & 1.2 \times 10^{-22} \\ 90 & 1.8 \times 10^{-22} \\ 95 & 2.8 \times 10^{-21} \\ 56 & 7.1 \times 10^{-20} \\ 127 & 1.2 \times 10^{-19} \\ 112 & 1.5 \times 10^{-19} \\ 100 & 2.0 \times 10^{-19} \\ 122 & 4.5 \times 10^{-19} \\ 192 & 4.7 \times 10^{-19} \\ 217 & 6.3 \times 10^{-19}\end{array}$

Figure 4 Enrichment analyses of common variants associated with intracranial volume. Enrichment of subsets of variants for association with intracranial volume by chromosomes (a), functional subtype (b) and pathway (c). See Online Methods for additional information. TFBS, transcription factor binding site; TSS, transcription start site; DGF, DNase genomic footprinting; DHS, DNase I hypersensitive sites; CTCF, CCCTC-binding factor. variant lying $14 \mathrm{~kb}$ downstream of HMGA2, and already showed suggestive association with intracranial volume in a previous report ${ }^{7}$. It has also previously been associated with height ${ }^{13}$ and is essential for growth $^{27}$. The chr10q24 LD-block covered multiple genes, but an intronic variant in PDCD11 was most significant. PDCD11 encodes an NF- $\kappa \mathrm{B}$-binding protein that is required for rRNA maturation and generation of $18 \mathrm{~S} \mathrm{rRNA}^{28}$. A variant in LD (rs7894407) has recently been identified in a GWAS of cerebral white matter hyperintensities $^{29}$. The top chr3q28 variant is located upstream of GMNC, which encodes the geminin coiled-coil domain-containing protein essential for DNA replication ${ }^{30}$.

Prior efforts to identify variants affecting intracranial volume were much smaller and did not adjust for height ${ }^{6-9}$. We found that four of seven loci had already been discovered for height ${ }^{13}$, and that over $10 \%$ of the known 'height loci' actually affected intracranial volume, even after regressing out height. Interestingly, some variants showed discordant associations for height and intracranial volume, consistent with the recent finding that different height loci disproportionally affect either leg length or spine and head length ${ }^{31}$, and may be a marker for pathological development ${ }^{32}$. In addition, height might therefore serve as a proxy phenotype for intracranial volume, with the tenfold larger sample of the height GWAS giving greater power to detect associations. Neural genes are also enriched in pathway analyses of height ${ }^{13}$. However, to fully disentangle whether these identified genes are 'height genes', 'brain volume genes' or 'growth genes' (that is, pleiotropic), a large collaborative effort is needed that examines the association of these variants with both intracranial volume and height in various models.

When investigating genome-wide overlap with other traits, we found a strong correlation with child head circumference, underlining the notion that intracranial volume is a valid measure for maximal attained brain size. We were able to leverage this genetic link by meta-analyzing both traits, which led to the identification of four additional loci (2q32.1, 3q23, 7p14.3 and 22q13.2). The correlations with birth length and weight were weaker and decreased further after adjusting for height, so a similar phenotypic correlation between head size and body size at younger age may drive these correlations. Intracranial volume was also genetically associated with cognitive function in childhood, as well as general cognitive function in middle-aged and older individuals. This indicates that variation in 

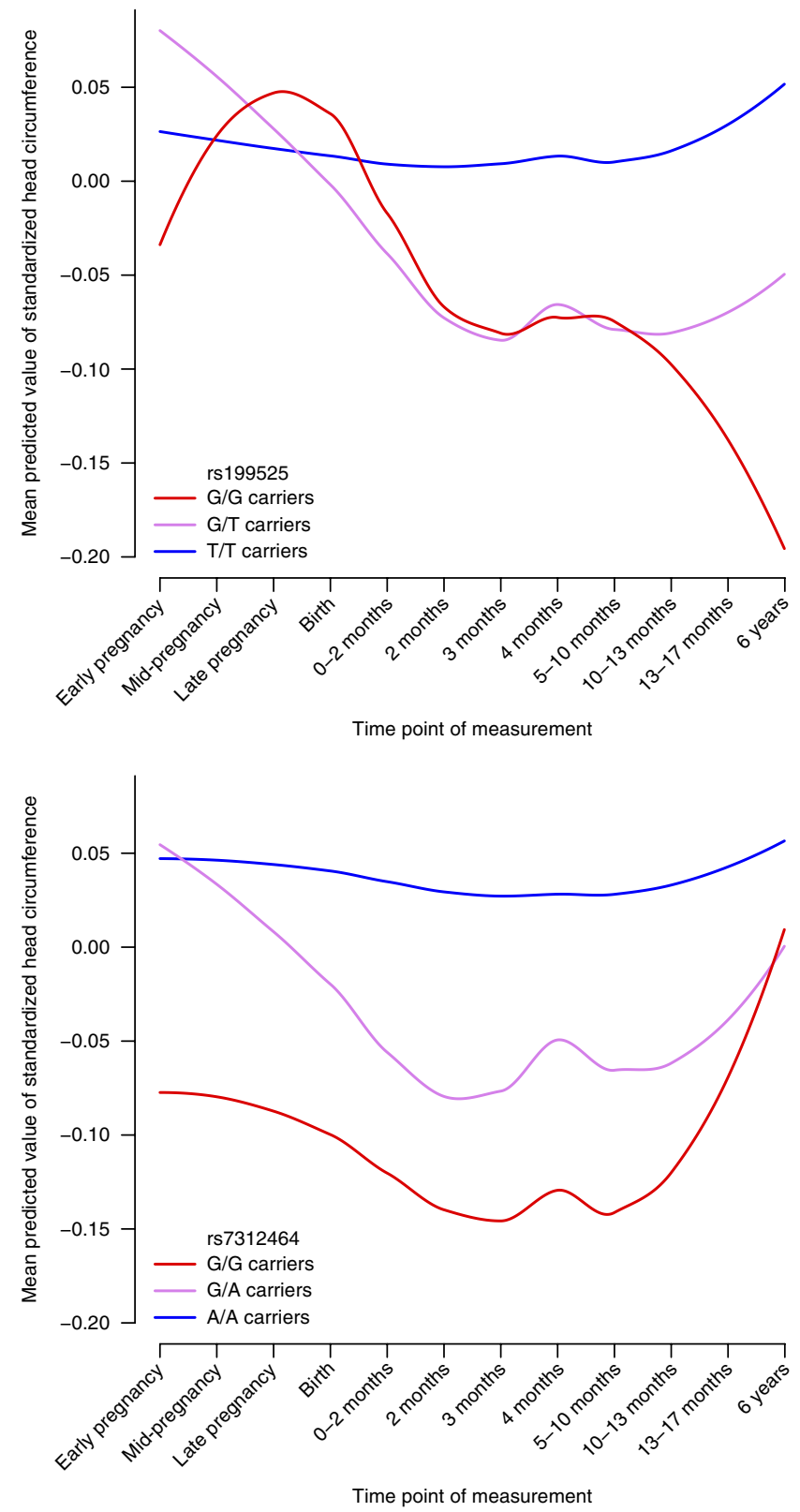

Figure 5 Temporal trends of intracranial volume loci during pre- and postnatal brain development. Mean predicted values of standardized head circumference using linear mixed models with age, sex, and the rs 199525 or rs 138074335 variants. The blue line represents children not carrying the risk allele, purple only a single risk allele and red with two risk alleles. See Online Methods for additional information. Total sample size was 2,824 .

maximally attained brain size during development shares a genetic basis with cognitive ability later in life and supports intracranial volume as a measure of brain reserve ${ }^{5}$.

The brain reserve hypothesis states that premorbid brain size can modify resilience to age-related brain pathology $y^{33}$, but there was no indication of a genome-wide overlap with Alzheimer's disease. However, we found a positive genetic correlation with Parkinson's disease, which instead points to a brain 'overgrowth' hypothesis. Notably, the IGF1 and the PI3K-AKT pathways, key factors in both growth signaling and our current study of intracranial volume, are neuroprotective in a model system of Parkinson's disease ${ }^{34}$. There were no correlations with other neurological or psychiatric traits, indicating that this finding might be specific to Parkinson's disease. However, it is important to note that there is a certain extent of variation in the sample size and power of these studies, and a larger GWAS might reveal genetic correlation with other traits as well. For schizophrenia, we also tested the latest GWAS (36,989 cases and 113,075 controls), but here too there was no significant correlation $\left(\rho_{\text {genetic }}=0.01, P=0.79\right)$.

It is not yet known whether variance in intracranial volume, in the normal range, contributes to disease risk or brain reserve. There is no doubt that in the pathological extremes of the distribution, size can matter, as in disorders such as microcephaly or macrocephaly. Here we found evidence for a shared genetic background between intracranial volume and cognitive function, and risk of Parkinson's disease. Although not definitive, these are new pieces of empirical evidence in the debate on whether or not whole brain size matters.

The pathway analyses highlight cellular growth and proliferation and included all components of the cell cycle (M, G1, S and G2 phases) and various growth factor signaling pathways. PI3K-AKT signaling has a well-described role in brain overgrowth disorders ${ }^{35,36}$, and was the only significant pathway using a different pathway analysis method (Supplementary Table 11). Interestingly, AKT3 intronic variants showed suggestive evidence for association with intracranial volume (rs7538011; $P=9.2 \times 10^{-7}$ ). Deletions of AKT3 cause microcephaly syndromes ${ }^{37}$, whereas duplications give rise to macrocephaly $^{38}$. Similar to FOXO3, it is part of the IGF1 signaling pathway, which is important for human longevity ${ }^{39}$. The PI3K-AKT signaling pathway seems to have an important role in brain growth, not only in pathological extremes, but also for normal variation at a population level. Other pathways enriched for association with intracranial volume highlight neuronal functions such as neurotransmission and axon guidance.

We identified previously unknown loci that influence intracranial volume, and, at a genome-wide level, there seemed to be common pathways, but our longitudinal study revealed that their developmental effects are complex. The loci influenced trajectories of head growth differently; it would also be interesting to investigate whether their spatial profiles of effects are distinct, such as certain loci promoting growth of particular brain regions.

Here we identified key genetic loci that have been implicated in intracranial volume in a global collaborative effort, followed by computational analyses to determine the important biological pathways and functional elements. Although the majority of the genetic variants are yet to be discovered, it is clear that these will provide better insight into brain development, as well as into related neuropsychiatric traits such as cognitive functioning and even for neurodegeneration late in life. Uncovering the remaining heritability will advance our understanding of the brain's complex genetic architecture.

URLs. LD score software: ftp://pricelab:pricelab@ftp.broadinstitute.org/LDSCORE/, ENIGMA protocols: http://enigma.usc. edu/protocols/genetics-protocols/, eQTL browser: http://genenetwork.nl/bloodeqtlbrowser/, LocusTrack software: http://gump.qimr. edu.au/general/gabrieC/LocusTrack/.

\section{METHODS}

Methods, including statements of data availability and any associated accession codes and references, are available in the online version of the paper.

Note: Any Supplementary Information and Source Data files are available in the online version of the paper. 


\section{ACKNOWLEDGMENTS}

CHARGE: Infrastructure for the CHARGE Consortium is supported in part by the National Heart, Lung and Blood Institute grant HL105756 and for the neuroCHARGE phenotype working group through the National Institute on Aging grant AG033193.

ENIGMA: ENIGMA was supported in part by a Consortium grant

(U54 EB020403 to PMT) from the NIH Institutes contributing to the Big Data

to Knowledge (BD2K) Initiative, including the NIBIB and NCI.

Age, Gene/Environment Susceptibility-Reykjavik Study (AGES-Reykjavik): This study has been funded by NIH contracts N01-AG-1-2100 and 271201200022C, the NIA Intramural Research Program, Hjartavernd (the Icelandic Heart Association), and the Althingi (the Icelandic Parliament). The study was approved by the Icelandic National Bioethics Committee, VSN: 00-063. The researchers are indebted to the participants for their willingness to participate in the study. Alzheimer's Disease Neuroimaging Initiative (ADNI): Data collection and sharing for this project was funded by the ADNI (National Institutes of Health Grant U01 AG024904) and DOD ADNI (Department of Defense award number W81XWH-12-2-0012). ADNI is funded by the National Institute on Aging, the National Institute of Biomedical Imaging and Bioengineering, and through generous contributions from the Alzheimer's Association; Alzheimer's Drug Discovery Foundation; BioClinica, Inc.; Biogen Idec Inc.; Bristol-Myers Squibb Company; Eisai Inc.; Elan Pharmaceuticals, Inc.; Eli Lilly and Company; F. Hoffmann-La Roche Ltd and its affiliated company Genentech, Inc.; GE Healthcare; Innogenetics, N.V.; IXICO Ltd.; Janssen Alzheimer Immunotherapy Research \& Development, LLC.; Johnson \& Johnson Pharmaceutical Research \& Development LLC.; Medpace, Inc.; Merck \& Co., Inc.; Meso Scale Diagnostics, LLC.; NeuroRx Research; Novartis Pharmaceuticals Corporation; Pfizer Inc.; Piramal Imaging; Servier; Synarc Inc.; and Takeda Pharmaceutical Company. The Canadian Institutes of Health Research provides funds to support ADNI clinical sites in Canada. Private sector contributions are facilitated by the Foundation for the National Institutes of Health (http://www.fnih.org). The grantee organization is the Northern California Institute for Research and Education, and the study is coordinated by the Alzheimer's Disease Cooperative Study at the University of California, San Diego. ADNI data are disseminated by the Laboratory of Neuro Imaging at the University of Southern California.

ANM: AddNeuroMed was funded through the EU FP6 programme. HS: Academy of Finland, Research Council for Health, 258081, UEFBrain, University of Eastern Finland, VTR funding Kuopio University Hospital.

Atherosclerosis Risk In Communities Study (ARIC): The Atherosclerosis Risk in Communities study was performed as a collaborative study supported by National Heart, Lung, and Blood Institute (NHLBI) contracts (HHSN268201100005C, HSN268201100006C, HSN268201100007C, HHSN268201100008C, HHSN268201100009C, HHSN268201100010C, HHSN268201100011C, and HHSN268201100012C), R01HL70825, R01HL087641, R01HL59367, and R01HL086694; National Human Genome Research Institute contract U01HG004402; and National Institutes of Health (NIH) contract HHSN268200625226C. Infrastructure was partly supported by grant no. UL1RR025005, a component of the NIH and NIH Roadmap for Medical Research. This project was also supported by NIH R01 grant NS087541 to M.F

Austrian Stroke Prevention Study Family (ASPS-Fam): The ASPS-Fam is funded by the Austrian Science Fund (FWF) project I904, the Medical University of Graz and the Steiermärkische Krankenanstalten Gesellschaft.

BETULA: This sample collection was supported by a Wallenberg Scholar grant from the Knut and Alice Wallenberg (KAW) foundation and a grant from Torsten and Ragnar Söderbergs Foundation to Lars Nyberg. S.1.H. was supported by a grant from HelseVest RHF (grant 911554).

Bipolar Family Study (BFS): The Bipolar Family Study wishes to thank the Scottish Mental Health Research Network for research assistant support, the Brain Research Imaging Centre Edinburgh, a center in the Scottish Funding Council Scottish Imaging Network-A Platform for Scientific Excellence (SINAPSE) Collaboration, for image acquisition and the Wellcome Trust Clinical Research Facility for genotyping. Genotyping was supported by the National Alliance for Research on Schizophrenia and Depression (NARSAD) Independent Investigator Award (to A.M.M.), and data collection was supported by the Health Foundation Clinician Scientist Fellowship. The research leading to these results also receives funding from the European Community's Seventh Framework Programme (FP7/2007- 2013) under grant agreements \#602450 (IMAGEMEND) and ongoing support from the Wellcome Trust (Ref 104036/Z/14/Z).

Brain Imaging Genetics (BIG): This work makes use of the BIG database, first established in Nijmegen, The Netherlands, in 2007. This resource is now part of Cognomics (http://www.cognomics.nl), a joint initiative by researchers of the Donders Centre for Cognitive Neuroimaging, the Human Genetics and Cognitive Neuroscience Departments of the Radboud University Medical Center and the Max Planck Institute for Psycholinguistics in Nijmegen. The Board of the
Cognomics Initiative consists of B. Franke, S. Fisher, G. Fernandez, P. Hagoort, H. Brunner, J. Buitelaar, H. van Bokhoven and D. Norris. The Cognomics Initiative has received supported from the participating departments and centers and from external grants, that is, the Biobanking and Biomolecular Resources Research Infrastructure (Netherlands) (BBMRI-NL), the Hersenstichting Nederland, and the Netherlands Organization for Scientific Research (NWO). The research leading to these results also receives funding from the NWO Gravitation grant 'Language in Interaction', the European Community's Seventh Framework Programme (FP7/2007-2013) under grant agreements no 602450 (IMAGEMEND), no 278948 (TACTICS), and no 602805 (Aggressotype), as well as from the European Community's Horizon 2020 programme under grant agreement no 643051 (MiND) and from ERC-2010-AdG 268800-NEUROSCHEMA. In addition, the work was supported by a grant for the ENIGMA Consortium (grant number U54 EB020403) from the BD2K Initiative of a cross-NIH partnership. We wish to thank all persons who kindly participated in the BIG research.

Brain Genomics Superstruct Project (GSP): Data were provided in part by the Brain Genomics Superstruct Project of Harvard University and the Massachusetts General Hospital, with support from the Center for Brain Science Neuroinformatics Research Group, the Athinoula A. Martinos Center for Biomedical Imaging, and the Center for Human Genetic Research. 20 individual investigators at Harvard and MGH generously contributed data to GSP. This work is supported by NIMH grants K99 MH101367 (P.H.L.), R01-MH079799 (J.W.S.), K24MH094614 (J.W.S.) and K01MH099232 (A.J.H.).

Brainscale and NTR-Adults: We would like to thank all twin participants from the Netherlands Twin Register. The NTR-adult and Brainscale studies were supported by the Netherlands Organization for Scientific Research NWO [MW904-61-193 (E.d.G. and D.B.), MaGW-nr: 400-07-080 (D. V‘t E.), MagW 480-04-004 (D.B.), (51.02.060 (H.H.), 668.772 (D.B. and H.H.); NWO/SPI 56-464-14192 (D.B.), the European Research Council (ERC-230374) (D.B.), High Potential Grant Utrecht University (H.H.), NWO Brain and Cognition 433-09-220 (H.H.) and the Neuroscience Campus Amsterdam.

Cardiovascular Health Study (CHS): This research was supported by NHLBI contracts HHSN268201200036C, HHSN268200800007C, N01HC55222, N01HC85079, N01HC85080, N01HC85081, N01HC85082, N01HC85083, N01HC85086; and NHLBI grants U01HL080295, R01HL087652, R01HL105756, R01HL103612, R01HL120393, and R01HL130114 with additional contribution from the National Institute of Neurological Disorders and Stroke (NINDS). Additional support was provided through R01AG023629, R01AG15928, R01AG20098, R01AG027002, R01AG05133, and R01AG027058 from the National Institute on Aging (NIA). A full list of principal CHS investigators and institutions can be found at http://CHS-NHLBI.org. The provision of genotyping data was supported in part by the National Center for Advancing Translational Sciences, CTSI grant UL1TR000124, and the National Institute of Diabetes and Digestive and Kidney Disease Diabetes Research Center (DRC) grant DK063491 to the Southern California Diabetes Endocrinology Research Center. The content is solely the responsibility of the authors and does not necessarily represent the official views of the National Institutes of Health.

CHAP: This research was funded by grants from the National Institute of Health (AG011101 and AG030146) and the International Alzheimer's Association (NIRP-14-302587). DNA samples were collected during clinical evaluations and population interviews, and analyzed at the Broad Institute.

Epidemiology of Dementia in Singapore (EDIS): The Singapore Malay Eye Study (SiMES) and the Singapore Chinese Eye. Study (SCES) are funded by National Medical Research Council (grants 0796/2003, IRG07nov013, IRG09nov014, STaR/0003/2008 and CG/SERI/2010) and Biomedical Research Council (grants 09/1/35/19/616), Singapore. The Genome Institute of Singapore, Agency for Science, Technology and Research, Singapore provided services for genotyping. The Epidemiology of Dementia in Singapore study is supported by the National Medical Research Council, Singapore (NMRC/CG/NUHS/2010, grant no. R-184-006-184-511). M.K.I. received additional funding from the Singapore Ministry of Health's National Medical Research Council (NMRC/CSA/038/2013).

EPIGEN: Work from the London Cohort was supported by research grants from the Wellcome Trust (grant 084730 to S.M.S.), University College London (UCL)/University College London Hospitals (UCLH) NIHR Biomedical Research Centre/Specialist Biomedical Research Centres (CBRC/SBRC) (grant 114 to S.M.S.), the European Union Marie Curie Reintegration (to M. Matarin and S.M.S.), the UK NIHR (08-08-SCC), the Comprehensive Local Research Network (CLRN) Flexibility and Sustainability Funding (FSF) (grant CEL1300 to S.M.S.), The Big Lottery Fund, the Wolfson Trust and the Epilepsy Society. This work was undertaken at UCLH/UCL, which received a proportion of funding from the UK Department of Health's NIHR Biomedical Research Centres funding scheme. Work from the Royal College of Surgeons in Ireland was supported by research grants from the Science Foundation Ireland (Research Frontiers Programme award 
08/RFP/GEN1538) and Brainwave-the Irish Epilepsy Association. M. Matarin is funded by Epilepsy Research UK (grant F1206).

Erasmus Rucphen Family study (ERF) The ERF study as a part of EUROSPAN (European Special Populations Research Network) was supported by European Commission FP6 STRP grant number 018947 (LSHG-CT-2006-01947) and also received funding from the European Community's Seventh Framework Programme (FP7/2007-2013)/grant agreement HEALTH-F4-2007-201413 by the European Commission under the programme "Quality of Life and Management of the Living Resources" of 5th Framework Programme (no. QLG2-CT-2002-01254). High-throughput analysis of the ERF data was supported by joint grant from Netherlands Organization for Scientific Research and the Russian Foundation for Basic Research (NWO-RFBR 047.017.043). We are grateful to all study participants and their relatives, general practitioners and neurologists for their contributions and to P. Veraart for her help in genealogy, J. Vergeer for the supervision of the laboratory work and P. Snijders for his help in data collection. N. Amin is supported by the Netherlands Brain Foundation (project number F2013(1)-28). The ERF study genome-wide array data and phenotype data (age and gender) is archived in European Genome-Phenome Database (EGA). The study is archived in the DAC named Erasmus Rucphen Family Study with the accession code: EGAS00001001134. Researchers who wish to use other phenotypic data of the Erasmus Rucphen Family Study must seek approval from the management team of the Erasmus Rucphen Family study. They are advised to contact Cornelia van Duijn (c.vanduijn@erasmusmc.nl).

Framingham Heart Study (FHS): This work was supported by the dedication of the Framingham Study participants, the National Heart, Lung and Blood Institute's Framingham Heart Study (Contract No. HHSN268201500001I), and by grants from the National Institute of Health (AG008122, AG054076, AG049607, AG033193, AG010129, NS017950, and U01AG49505).

Generation R: The Generation R Study is conducted by the Erasmus Medical Centre in close collaboration with the Municipal Health Service Rotterdam area, and the Stichting Trombosedienst \& Artsenlaboratorium Rijnmond (STAR), Rotterdam. We gratefully acknowledge the contribution of general practitioners, hospitals, midwives and pharmacies in Rotterdam. Additional support for neuroimaging came from ZonMw TOP 40-00812-98-11021.

GeneSTAR: GeneSTAR was supported by grants from the National Institute of Neurological Disorders and Stroke (R01NS062059), the National Heart, Lung, and Blood Institute (U01 HL72518, HL097698) and the National Institutes of Health/National Center for Research Resources (M01-RR000052) to the Johns Hopkins General Clinical Research Center. We would like to thank the participants and families of GeneSTAR and our dedicated staff for all their sacrifices. GIG: The GIG (Genomic Imaging Göttingen) sample was established at the Center for Translational Research in Systems Neuroscience and Psychiatry at Göttingen University. We thank M. Keil, E. Diekhof, T. Melcher and I. Henseler for assistance in MRI data acquisition, and E. Binder and H. Mohr for their valuable help with genotyping. We are grateful to all persons who kindly participated in the GIG study.

GOBS: We acknowledge the ultimate source of our data, the Mexican American community of San Antonio and surrounding areas. Financial support for this study was provided by grants from the National Institute of Mental Health MH0708143 (D.C. Glahn), MH078111 (J. Blangero) and MH083824 (D.C. Glahn and J. Blangero). Theoretical development of SOLAR is supported by MH59490 (J. Blangero). This investigation was conducted, in part, in facilities constructed with support from Research Facilities Improvement Program grant numbers C06 RR13556 and C06 RR017515 from the National Center for Research Resources, NIH. Some of this work was performed at Texas Biomedical Research Institute, where J. Blangero began this investigator-initiated competitively publicly funded work.

HUBIN: This study was financed by the Swedish Research Council

(K2007-62X-15077-04-1, K2008-62P-20597-01-3. K2010-62X-15078-07-2, K2012-61X-15078-09-3), the regional agreement on medical training and clinical research between Stockholm County Council and the Karolinska Institutet, the Knut and Alice Wallenberg Foundation, and the HUBIN project. Genotyping was performed by the SNP\&SEQ Technology Platform in Uppsala. The platform is part of Science for Life Laboratory at Uppsala University and supported as a national infrastructure by the Swedish Research Council.

IMAGEN: IMAGEN was supported by the European Union-funded FP6 Integrated Project IMAGEN (Reinforcement-related behavior in normal brain function and psychopathology) (LSHM-CT- 2007-037286), the FP7 projects IMAGEMEND (602450) and MATRICS (603016), and the Innovative Medicine Initiative Project EU-AIMS (115300-2), the Medical Research Council Programme Grant "Developmental pathways into adolescent substance abuse" (93558), as well as the NIHR-biomedical Research Center "Mental Health". Further support was provided by the Swedish Research Council FORMAS, and the German Federal Ministry for Education and Research BMBF (eMED
SysAlc 01ZX1311A; Forschungsnetz AERIAL; 1EV0711) and the US National Institutes of Health (Axon, Testosterone and Mental Health during Adolescence; MH085772-01A1), and grants from the French MILDECA and from the Fondation pour la Recherche Médicale.

IMpACT: This study was funded by a grant from the Brain \& Cognition Excellence Program of the Netherlands Organization for Scientific Research (NWO, grant 433-09-229) and in part by the Netherlands Brain Foundation (grant number, 15F07 [2]27). B. Franke is supported by a Vici grant from the Netherlands Organization for Scientific Research (NWO; grant $\mathrm{n}^{\circ}$ 016.130.669). The research leading to these results also receives funding from the European Community's Seventh Framework Programme (FP7/2007-2013) under grant agreements n ${ }^{\circ} 602450$ (IMAGEMEND), n²78948 (TACTICS), and nº602805 (Aggressotype) as well as from the European Community's Horizon 2020 programme under grant agreement $n^{\circ} 643051$ (MiND). In addition, the work was supported by a grant for the ENIGMA Consortium (grant number U54 EB020403) from the BD2K Initiative of a cross-NIH partnership.

LBC1936: We thank the LBC1936 participants and the members of the LBC1936 research team who collected and collated the phenotypic and genotypic data. This work was undertaken as part of the Cross Council and University of Edinburgh Centre for Cognitive Ageing and Cognitive Epidemiology (CCACE; http://www. ccace.ed.ac.uk). This work was supported by a Research into Ageing programme grant (to I.J.D.) and the Age UK-funded Disconnected Mind project (http://www. disconnectedmind.ed.ac.uk; to I.J.D. and J.M.W.), with additional funding from the UK Medical Research Council (MRC; to I.J.D., J.M.W. and M.E.B.). J.M.W. is supported by the Scottish Funding Council through the SINAPSE Collaboration (http://www.sinapse.ac.uk). M.V.M. is supported by the Row Fogo Charitable Trust. CCACE (MRC MR/K026992/1) is funded by the UK Biotechnology and Biological Sciences Research Council (BBSRC) and the UK MRC. Genotyping was supported by a grant from the BBSRC (BB/F019394/1).The image acquisition and analysis was performed at the Brain Research Imaging Centre, University of Edinburgh (http://www.bric.ed.ac.uk).

Leiden Longevity Study (LLS): The Leiden Longevity Study was supported by a grant from the Innovation-Oriented Research Program on Genomics (SenterNovem IGE05007) and the Netherlands Consortium for Healthy Ageing (grant number 050-060-810).

Mind Clinical Imaging Consortium (MCIC): Data used in the preparation of this work were obtained from the Mind Clinical Imaging Consortium database through the Mind Research Network (http://www.mrn.org). The MCIC project was supported by the Department of Energy under Award Number DE-FG02-08ER64581. MCIC is the result of efforts of co-investigators from University of Iowa, University of Minnesota, University of New Mexico, and Massachusetts General Hospital.

MooDS: The establishment of the MooDS sample was funded by the German Federal Ministry of Education and Research (BMBF) through the Integrated Genome Research Network (IG) MooDS (Systematic Investigation of the Molecular Causes of Major Mood Disorders and Schizophrenia; grant 01GS08144 to M.M. Nöthen and S. Cichon, grant 01GS08147 to J. Rietschel and A. MeyerLindenberg and grant 01GS08148 to A. Heinz), under the auspices of the National Genome Research Network plus (NGFNplus), and through the Integrated Network IntegraMent (Integrated Understanding of Causes and Mechanisms in Mental Disorders), under the auspices of the e:Med Programme (grant 01ZX1314A to M.M. Nöthen, grant 01ZX1314C to H. Walter, grant 01ZX1314G to M. Rietschel). MPIP: The MPIP Munich Morphometry Sample comprises images acquired as part of the Munich Antidepressant Response Signature Study and the Recurrent Unipolar Depression (RUD) Case-Control study performed at the MPIP, and control subjects acquired at the Ludwig-Maximilians-University, Munich, Department of Psychiatry. We thank E. Meisenzahl and D. Rujescu for providing MRI and genetic data for inclusion into the MPIP Munich Morphometry sample. We wish to acknowledge A. Olynyik and radiographers R. Schirmer, E. Schreiter, R. Borschke and I. Eidner for image acquisition and data preparation. We thank D.P. Auer for local study management in the initial phase of the RUD study. We are grateful to GlaxoSmithKline for providing the genotypes of the Recurrent Unipolar Depression Case-Control Sample. We thank the staff of the Center of Applied Genotyping (CAGT) for generating the genotypes of the MARS cohort. The study is supported by a grant of the Exzellenz-Stiftung of the Max Planck Society. This work has also been funded by the Federal Ministry of Education and Research (BMBF) in the framework of the National Genome Research Network (NGFN), FKZ 01GS0481.

NCNG: this sample collection was supported by grants from the Bergen Research Foundation and the University of Bergen, the Dr. Einar Martens Fund, the K.G. Jebsen Foundation, the Research Council of Norway, to S.L.H., V.M.S. and T.E. NESDA: Funding was obtained from the Netherlands Organization for Scientific Research (Geestkracht program grant 10-000-1002); the Center for Medical Systems Biology (CSMB, NWO Genomics), Biobanking and Biomolecular 
Resources Research Infrastructure (BBMRI-NL), VU University's Institutes for Health and Care Research (EMGO+) and Neuroscience Campus Amsterdam, University Medical Center Groningen, Leiden University Medical Center, National Institutes of Health (NIH, R01D0042157-01A, MH081802, Grand Opportunity grants 1RC2 MH089951 and 1RC2 MH089995). Part of the genotyping and analyses were funded by the Genetic Association Information Network (GAIN) of the Foundation for the National Institutes of Health. Computing was supported by BiG Grid, the Dutch e-Science Grid, which is financially supported by NWO. NeuroIMAGE: The NeuroIMAGE project was supported by NIH Grant R01MH62873 (to S.V. Faraone), NWO Large Investment Grant 1750102007010 (to J. Buitelaar), and by grants from Radboud University Nijmegen Medical Center, University Medical Center Groningen and Accare, and VU University Amsterdam. The work contributing to this result also receives support from the European Community's Seventh Framework Programme (FP7/2007- 2013) under grant agreements $\mathrm{n}^{\circ} 602450$ (IMAGEMEND), $\mathrm{n}^{\circ} 278948$ (TACTICS) and $\mathrm{n}^{\circ} 602805$ (Aggressotype) as well as from the European Community's Horizon 2020 programme under grant agreement $\mathrm{n}^{\circ} 643051$ (MiND). In addition, the work was supported by a grant for the ENIGMA Consortium (grant number U54 EB020403) from the BD2K Initiative of a cross-NIH partnership.

NIMH-IRP: Supported in part by the NIMH Intramural Research Program (ZIAMH002810; Z01MH002792; Z01MH002790).

North American Brain Expression Consortium (NABEC): This research was supported by the Intramural Research Program of the NIH, National Institute on Aging.

Older Australian Twins Study (OATS): We would like to acknowledge and thank the OATS participants, their supporters and respective Research Teams. This work was supported by a number of sources. OATS is supported by the NHMRC/Australian Research Council Strategic Award 401162 and NHMRC Project Grant 1045325 to P. Sachdev and colleagues. OATS was facilitated through access to the Australian Twin Registry, a national research resource supported by the NHMRC Enabling Grant 310667, administered by the University of Melbourne. DNA was extracted by Genetic Repositories Australia, an Enabling Facility supported by the NHMRC Grant 401184. OATS genotyping was partly funded by a Commonwealth Scientific and Industrial Research Organisation Flagship Collaboration Fund Grant. Henry Brodaty is supported by the Australian Government funded Dementia Collaborative Research Centre (DCRC), UNSW. Nicola Armstrong was supported by the NHMRC Project Grant 525453 and Karen Mather is supported by an Alzheimer's Australia Dementia Research Foundation Postdoctoral Fellowship and the NHMRC Capacity Building Grant 568940.

Osaka: This study was supported, in part, by research grants from the Japanese Ministry of Health, Labor and Welfare; the Japanese Ministry of Education, Culture, Sports, Science and Technology (MEXT) KAKENHI and Scientific Research on Innovative Areas (Comprehensive Brain Science Network); and the Brain Sciences Project of the Center for Novel Science Initiatives (CNSI), the National Institutes of Natural Sciences (NINS), and the Brain Mapping by Integrated Neurotechnologies for Disease Studies (Brain/MINDS) from Japan Agency for Medical Research and development, AMED.

PAFIP: The PAFIP study was supported by Instituto de Salud Carlos III, FIS00/3095, 01/3129, PI020499, PI060507, PI10/00183, PI14/00639, the SENY Fundació Research Grant CI 2005-0308007, and the Fundación Marqués de Valdecilla API07/011. PAFIP wish to acknowledge WTCCC2 (Wellcome Trust Case Control Consortium 2) for DNA Genotyping, Valdecilla Biobank for providing the biological samples and associated data included in this study and Idival Neuroimaging Unit for its help in the technical execution of this work. D. Tordesillas-Gutiérrez is funded by a contract from the Carlos III Health Institute (CA12/00312).

PROSPER: The PROSPER study was supported by an investigator-initiated grant obtained from Bristol-Myers Squibb. J.W. Jukema is an Established Clinical Investigator of the Netherlands Heart Foundation (grant 2001 D 032). Support for genotyping was provided by the seventh framework program of the European commission (grant 223004) and by the Netherlands Genomics Initiative (Netherlands Consortium for Healthy Aging grant 050-060-810).

QTIM: D.P.H., N.J., C.R.K.C. and P.M.T. are supported, in part, by NIH grants R01 NS080655, R01AG040060, R01 EB008432, R01 MH097268, U01 AG024904, R01 MH085667, R01 MH089722, P41 EB015922, and R01 MH094343. R.K.W. is supported by National Science Foundation (BCS-1229450). J.L.S. was supported by the NIMH (K99MH102357) and Autism Speaks. G.Z. is supported by a Future Fellowship (FT0991634) from the Australian Research Council. S.E.M. and G.W.M. are supported by a National Health and Medical Research Council (NHMRC), Australia, Fellowships $(1103623,619667)$. The QTIM study is supported by grants from NIH (R01 HD050735) and the NHMRC (389875, 486682, 1009064). We thank the twins and siblings for their participation, M. Grace and A. Eldridge for twin recruitment, A. Al Najjar and other radiographers for scanning,
K. McAloney and D. Park for research support, and A. Henders and staff for DNA sample processing and preparation.

ROS and MAP: The clinical, genomic, and neuroimaging data for the Religious Orders Study and the Rush Memory and Aging Project was funded by NIH grants P30AG10161, RF1AG15819, R01AG17917, R01AG30146, R01AG40039, and the Translational Genomics Research Institute.

Rotterdam Study: The generation and management of GWAS genotype data for the Rotterdam Study are supported by the Netherlands Organisation of Scientific Research NWO Investments (nr. 175.010.2005.011, 911-03-012). This study is funded by the Research Institute for Diseases in the Elderly (014-93-015; RIDE2), the Netherlands Genomics Initiative (NGI)/Netherlands Organisation for Scientific Research (NWO) project nr. 050-060-810. The Rotterdam Study is funded by Erasmus Medical Center and Erasmus University, Rotterdam, Netherlands Organization for the Health Research and Development (ZonMw), the Research Institute for Diseases in the Elderly (RIDE), the Ministry of Education, Culture and Science, the Ministry for Health, Welfare and Sports, the European Commission (DG XII), and the Municipality of Rotterdam. This research is supported by the Dutch Technology Foundation STW, which is part of the NWO, and which is partly funded by the Ministry of Economic Affairs. This project has received funding from the European Research Council (ERC) under the European Union's Horizon 2020 research and innovation programme (grant agreement No 678543). Further support was obtained through the Joint Programme - Neurodegenerative Disease Research working group on High-Dimensional Research in Alzheimer's Disease (ZonMW grant number 733051031). MAI is supported by ZonMW grant number 916.13.054. H.H.H.A. is supported by the Van Leersum Grant of the Royal Netherlands Academy of Arts and Sciences. Saguenay Youth Study (SYS): The Saguenay Youth Study project is funded by the Canadian Institutes of Health Research (T.P., Z.P.), Heart and Stroke Foundation of Quebec (Z.P.), and the Canadian Foundation for Innovation (Z.P.). T.P. is supported by the Tanenbaum Chair in Population Neuroscience at the Rotman Research Institute, University of Toronto.

SHIP and TREND: The SHIP data sets are part of the Community Medicine Research net (CMR) of the University of Greifswald, which is funded by the German Federal Ministry of Education and Research and the German Ministry of Cultural Affairs, as well as by the Social Ministry of the Federal State of Mecklenburg-West Pomerania (grants no. 01ZZ9603, 01ZZ0103, and 01ZZ0403), and the network 'Greifswald Approach to Individualized Medicine (GANI_MED)' funded by the Federal Ministry of Education and Research (grant 03IS2061A). Genome-wide data and MRI scans were supported by the Federal Ministry of Education and Research (grant no. 03ZIK012) and a joint grant from Siemens Healthcare, Erlangen, Germany, and the Federal State of Mecklenburg-West Pomerania. The University of Greifswald is a member of $t$ the Caché Campus Program of the InterSystems GmbH.

Sydney Memory and Ageing Study (Sydney MAS): We would like to thank the Sydney MAS participants, their supporters and respective research teams. Sydney MAS was supported by the Australian National Health and Medical Research Council (NHMRC) Program Grants 350833 and 568969 to P. Sachdev, H. Brodaty and G. Andrews. DNA was extracted by Genetic Repositories Australia, an Enabling Facility supported by the NHMRC Grant 401184 . H. Brodaty is supported by the Australian Government funded Dementia Collaborative Research Centre (DCRC), UNSW. N. Armstrong was supported by the NHMRC Project Grant 525453 and K. Mather is supported by an Alzheimer's Australia Dementia Research Foundation Postdoctoral Fellowship. Both S. Reppermund and K. Mather are supported by the NHMRC Capacity Building Grant 568940.

Tasmanian Study of Gait and Cognition (TASCOG): The Tasmanian Study of Gait and Cognition is supported by project grants from the National Health and Medical Research Council of Australia (NHMRC; 403000,491109, and 606543) and a grant from the Wicking Dementia Education and Research Centre, Hobart. V.S. is supported by a cofunded NHMRC Career Development Fellowship (1061457) and a Heart Foundation Future Leader Fellowship (ID 100089).

Three-City Dijon Study: The Three-City Study is conducted under a partnership agreement among the Institut National de la Santé et de la Recherche Médicale (INSERM), the Victor Segalen-Bordeaux II University, and Sanofi-Aventis. The Fondation pour la Recherche Médicale funded the preparation and initiation of the study. The Three-City Study is also supported by the Caisse Nationale Maladie des Travailleurs Salariés, Direction Générale de la Santé, Mutuelle Générale de l'Education Nationale (MGEN), Institut de la Longévité, Conseils Régionaux of Aquitaine and Bourgogne, Fondation de France, and Ministry of ResearchINSERM Programme "Cohortes et collections de données biologiques." Christophe Tzourio and Stéphanie Debette are supported by a grant from the Fondation Leducq.

TOP: The study was supported by the Research Council of Norway ( $\# 213837$, \#223273, \#229129), South-East Norway Health Authority (\#2013-123) and KG Jebsen Foundation. The research leading to these results has received 
funding from the European Community's Seventh Framework Programme (FP7/2007-2013) under grant agreement $n^{\circ} 602450$ (IMAGEMEND).

UCLA_NL_BP: Data collection and genotyping was made possible with (NIH/NIMH) R01 MH090553 to R.A.O.th-East Norway Health Authority (\#2013-123) and KG Jebsen Foundation.

UMCU: UMCU acknowledgment data: This work was supported by 917.46 .370 (H.H.) and 908-02-123 (H.H.) from the Netherlands Organization for Health Research and Development ZonMW.

WHICAP: This study was supported by a grant from the NIH (5R01AG037212). CHARGE consortium: See ref. 40 for the general cognitive function GWAS, and ref. 29 for the white matter lesion GWAS.

Early Growth Genetics (EGG) consortium: Data on head circumference, birth weight, and birth length have been contributed by the EGG Consortium and was downloaded from http://www.egg-consortium.org.

Genetic Investigation of ANthropometric Traits (GIANT) consortium:

See ref. 13.

Genetics of Personality Consortium: See ref. 41 for the neuroticism GWAS and ref. 42 for the extraversion GWAS.

IGAP: We thank the International Genomics of Alzheimer's Project (IGAP) for providing summary results data for these analyses. The investigators within IGAP contributed to the design and implementation of IGAP and/or provided data but did not participate in analysis or writing of this report. IGAP was made possible by the generous participation of the control subjects, the patients, and their families. The i-Select chips was funded by the French National Foundation on Alzheimer's disease and related disorders. EADI was supported by the LABEX (laboratory of excellence program investment for the future) DISTALZ grant, Inserm, Institut Pasteur de Lille, Université de Lille 2 and the Lille University Hospital. GERAD was supported by the Medical Research Council (Grant $n^{\circ} 503480$ ), Alzheimer's Research UK (Grant $n^{\circ}$ 503176), the Wellcome Trust (Grant n $\left.{ }^{\circ} 082604 / 2 / 07 / Z\right)$ and German Federal Ministry of Education and Research (BMBF): Competence Network Dementia (CND) grant n ${ }^{\circ}$ 01GI0102, 01GI0711, 01GI0420. CHARGE was partly supported by the NIH/NIA grant R01 AG033193 and the NIA AG081220 and AGES contract N01-AG-12100, the NHLBI grant R01 HL105756, the Icelandic Heart Association, and the Erasmus Medical Center and Erasmus University. ADGC was supported by the NIH/NIA grants: U01 AG032984, U24 AG021886, U01 AG016976, and the Alzheimer's Association grant ADGC-10-196728.

International Parkinson's Disease Genomics Consortium (IPDGC): See ref. 43. Psychiatric Genomics Consortium: See ref. 44.

Social Science Genetic Association Consortium (SSGAC): See ref. 45 for the childhood cognitive function GWAS.

\section{AUTHOR CONTRIBUTIONS}

Conceived of the study and drafted the manuscript: H.H.H.A., D.P.H., V.C., J.L.S., M.E.R., S.T., A.A., P.N, V.G., G.S., M.F., B.F., S.D., S.E.M., M.A.I., P.M.T. Performed statistical analyses: H.H.H.A., D.P.H., V.C., J.L.S., M.E.R., S.T., A.A., Sy.D., A.H.B., N.J., K.W., Lu.A., N.A., M.A., B.S.A., N.J.A., La.A., A.B., M.B., J.C.B., L.M.E.B., S.H.B., M.M.B., Ja.B., O.C., M.M.C., Ga.C., Q.C., C.R.K.C., G.C., Nh.D., St.E., Ti.G., Su.G., A.L.G., C.U.G., Ol.G., M.E.G., T.G., Jo.H., U.K.H., S.H., E.H., M.H., D.J., T.J., N.K., D.K., S.K., M.K., B.K., P.H.L., J.L., D.C.M.L., L.M.L., M.L., Ch.M., Su.M., An.M., Ma.M., M.M., Be.M., D.R.M., R.M., Y.M., R.L.M., K.N., L.M.O., J.O., Ma.P., I.P., L.P., S.P., B.P., K.B.R., A.R., J.S.R., S.L.R., R.R., Na.R., N.A.R., T.R., C.L.S., Li.S., An.J.S., L.S., J.S., A.V.S., E.S., L.T.S., Al.T., Ro.T., Di.T., R.T., D.T., Dh.V., J.V., S.J.V., D.vdM., M.M.J.V., K.R.V., D.vR., Es.W., L.T.W., A.M.W., G.W., C.W., Th.W., L.R.Y., J.Y., M.P.Z., A.M.D., I.O.F., B.M., T.E.N., J.A.T., B.X., Sa.A., A.M.B., A.dB., A.J.H., A.C.N., P.G.S., C.D.W., S.M.B., R.M.B., G.D., J.G., O.G., R.K., C.M., M.A.N., D.V., B.N.V., T.W., E.J.R. Acquired data: P.N., Su.S., K.A.A., T.A., M.P.B., Ir.F., R.F.G., D.H., K.A.M., Em.S., B.G.W., A.Z., I.A., N.T.A., L.A., D.A., P.A., O.A.A., S.A., A.A.A., M.E.B., D.M.B., J.T.B., D.A.B., J.B., H.v.a.B., D.I.B., H.B., H.G.B., R.L.B., J.K.B., K.B.B., W.C., V.D.C., D.M.C., G.L.C., C-Y.C., C.C., S.C., M.R.C., A.C., B.C., J.E.C., M.C., G.E.D., E.J.C.D., P.L.D., G.I.D., N.D., Ch.D., A.DeS., A.D., Sr.D., W.C.D., R.D., T.D.D., S.E., T.E., D.A.E., G.F., L.F., S.E.F., D.A.F., I.F., T.M.F., P.T.F., C.F., Ma.F., D.C.G., R.L.G., H.H.H.G., H.J.G., R.C.G., S.G., N.K.H., J.H., C.A.H., R.H., K.H., An.H., S.L.e.H., D.G.H., D.J.H., B.H., P.J.H., W.H., A.H., F.H., G.H., N.H., J-J.H., H.E.H., M.I., M.K.I., C.R.J., R.J., E.G.J., J.J., R.S.K., I.K., D.S.K., P.K., J.B.K., L.J.L., S.M.L., H.L., X.L., D.L.L., W.L., O.L.L., S.L., O.M., J.M., V.S.M., A.M.M., F.J.M., K.L.M., P.M., I.M., A.M., S.M., G.W.M., D.W.M., T.H.M., T.W.M., M.N., W.J.N., M.M.N., L.N., K.O., R.L.O., R.A.O., M.P., T.P., Z.P., B.W.J.P., G.P., S.G.P., B.M.P., S.R., Ma.R., J.L.R., N.R., J.I.R., M.R., R.L.S., P.S.S., A.J.S., He.S., P.R.S., S.S., A.S., S.M.S., C.S., J.W.S., H.S., V.S., V.M.S., D.J.S., J.E.S., A.T., H.T., A.W.T., B.T., J.T., C.T., A.G.U., M.C.V., M.vdB., A.V., N.J.A.V., C.M.V., N.E.M.V., M.V., D.J.V., M.W.V., H.V., H.W., J.M.W., T.H.W., M.E.W., D.R.W., M.W.W., W.W., E.W., T.Y.W., C.B.W., R.H.Z., A.B.Z., I.J.D., C.D., R.S., N.G.M., A.J.M.D., M.J.W., V.G., G.S., M.F., B.F., S.D., S.E.M., M.A.I., P.M.T., A.Sim.,
Sa.A., A.M.B., A.dB., A.J.H., A.C.N., P.G.S., C.D.W., S.M.B., R.M.B., G.D., J.G., O.G., R.K., C.M., M.A.N., D.V., B.N.V., T.W., E.J.R. All authors critically reviewed the manuscript for important intellectual content.

\section{COMPETING FINANCIAL INTERESTS}

The authors declare no competing financial interests.

Reprints and permissions information is available online at http://www.nature.com/ reprints/index.html.

1. Bartley, A.J., Jones, D.W. \& Weinberger, D.R. Genetic variability of human brain size and cortical gyral patterns. Brain 120, 257-269 (1997).

2. Davis, P.J.M. \& Wright, E.A. A new method for measuring cranial cavity volume and its application to the assessment of cerebral atrophy at autopsy. Neuropathol. Appl. Neurobiol. 3, 341-358 (1977).

3. Sgouros, S., Goldin, J.H., Hockley, A.D., Wake, M.J. \& Natarajan, K. Intracranial volume change in childhood. J. Neurosurg. 91, 610-616 (1999).

4. Buckner, R.L. et al. A unified approach for morphometric and functional data analysis in young, old and demented adults using automated atlas-based head size normalization: reliability and validation against manual measurement of total intracranial volume. Neuroimage 23, 724-738 (2004).

5. Farias, S.T. et al. Maximal brain size remains an important predictor of cognition in old age, independent of current brain pathology. Neurobiol. Aging 33 , 1758-1768 (2012)

6. Ikram, M.A. et al. Common variants at $6 \mathrm{q} 22$ and $17 \mathrm{q} 21$ are associated with intracranial volume. Nat. Genet. 44, 539-544 (2012).

7. Stein, J.L. et al. Identification of common variants associated with human hippocampal and intracranial volumes. Nat. Genet. 44, 552-561 (2012).

8. Hibar, D.P. et al. Common genetic variants influence human subcortical brain structures. Nature 520, 224-229 (2015).

9. Paus, T. et al. KCTD8 gene and brain growth in adverse intrauterine environment: a genome-wide association study. Cereb. Cortex 22, 2634-2642 (2012).

10. Psaty, B.M. et al. Cohorts for Heart and Aging Research in Genomic Epidemiology (CHARGE) Consortium: design of prospective meta-analyses of genome-wide association studies from 5 cohorts. Circ Cardiovasc Genet 2, 73-80 (2009).

11. Thompson, P.M. et al. The ENIGMA Consortium: large-scale collaborative analyses of neuroimaging and genetic data. Brain Imaging Behav. 8, 153-182 (2014).

12. Bulik-Sullivan, B.K. et al. LD Score regression distinguishes confounding from polygenicity in genome-wide association studies. Nat. Genet. 47, 291-295 (2015).

13. Wood, A.R. et al. Defining the role of common variation in the genomic and biological architecture of adult human height. Nat. Genet. 46, 1173-1186 (2014).

14. Stefansson, H. et al. A common inversion under selection in Europeans. Nat. Genet. 37, 129-137 (2005).

15. Spillantini, M.G. \& Goedert, M. Tau pathology and neurodegeneration. Lancet Neurol. 12, 609-622 (2013).

16. Desikan, R.S. et al. Genetic overlap between Alzheimer's disease and Parkinson's disease at the MAPT locus. Mol. Psychiatry 20, 1588-1595 (2015).

17. Kirchhoff, M., Bisgaard, A.M., Duno, M., Hansen, F.J. \& Schwartz, M. A 17q21.31 microduplication, reciprocal to the newly described $17 q 21.31$ microdeletion, in a girl with severe psychomotor developmental delay and dysmorphic craniofacial features. Eur. J. Med. Genet. 50, 256-263 (2007).

18. Koolen, D.A. et al. Mutations in the chromatin modifier gene KANSL1 cause the 17q21.31 microdeletion syndrome. Nat. Genet. 44, 639-641 (2012).

19. Estrada, K. et al. Genome-wide meta-analysis identifies 56 bone mineral density loci and reveals 14 loci associated with risk of fracture. Nat. Genet. 44, 491-501 (2012).

20. Kemp, J.P. et al. Phenotypic dissection of bone mineral density reveals skeletal site specificity and facilitates the identification of novel loci in the genetic regulation of bone mass attainment. PLoS Genet. 10, e1004423 (2014).

21. Broer, L. et al. GWAS of longevity in CHARGE consortium confirms $A P O E$ and FOXO3 candidacy. J. Gerontol. A Biol. Sci. Med. Sci. 70, 110-118 (2015).

22. Kaplan, R.C. et al. A genome-wide association study identifies novel loci associated with circulating IGF-I and IGFBP-3. Hum. Mol. Genet. 20, 1241-1251 (2011).

23. Paik, J.H. et al. FoxOs cooperatively regulate diverse pathways governing neural stem cell homeostasis. Cell Stem Cell 5, 540-553 (2009).

24. Renault, V.M. et al. FoxO3 regulates neural stem cell homeostasis. Cell Stem Cell 5, 527-539 (2009)

25. Rzehak, P. et al. Associations of IGF-1 gene variants and milk protein intake with IGF-I concentrations in infants at age 6 months: results from a randomized clinical trial. Growth Horm. IGF Res. 23, 149-158 (2013).

26. Taal, H.R. et al. Common variants at $12 \mathrm{q} 15$ and $12 \mathrm{q} 24$ are associated with infant head circumference. Nat. Genet. 44, 532-538 (2012).

27. Lynch, S.A. et al. The $12 \mathrm{q} 14$ microdeletion syndrome: six new cases confirming the role of HMGA2 in growth. Eur. J. Hum. Genet. 19, 534-539 (2011).

28. Sweet, T., Yen, W., Khalili, K. \& Amini, S. Evidence for involvement of NFBP in processing of ribosomal RNA. J. Cell. Physiol. 214, 381-388 (2008).

29. Verhaaren, B.F. et al. Multi-ethnic genome-wide association study of cerebral white matter hyperintensities on MRI. Circgenetics. 114, 000858 (2015).

30. Balestrini, A., Cosentino, C., Errico, A., Garner, E. \& Costanzo, V. GEMC1 is a TopBP1-interacting protein required for chromosomal DNA replication. Nat. Cell Biol. 12, 484-491 (2010). 
31. Chan, Y. et al. Genome-wide analysis of body proportion classifies height-associated variants by mechanism of action and implicates genes important for skeletal development. Am. J. Hum. Genet. 96, 695-708 (2015).

32. Fukumoto, A. et al. Head circumference and body growth in autism spectrum disorders. Brain Dev. 33, 569-575 (2011).

33. Stern, Y. Cognitive reserve in ageing and Alzheimer's disease. Lancet Neurol. 11, 1006-1012 (2012).

34. Quesada, A., Lee, B.Y. \& Micevych, P.E. PI3 kinase/Akt activation mediates estrogen and IGF-1 nigral DA neuronal neuroprotection against a unilateral rat model of Parkinson's disease. Dev. Neurobiol. 68, 632-644 (2008).

35. Hevner, R.F. Brain overgrowth in disorders of RTK-PI3K-AKT signaling: a mosaic of malformations. Semin. Perinatol. 39, 36-43 (2015).

36. Rivière, J.-B. et al. De novo germline and postzygotic mutations in AKT3, PIK3R2 and PIK3CA cause a spectrum of related megalencephaly syndromes. Nat. Genet. 44, 934-940 (2012).

37. Boland, E. et al. Mapping of deletion and translocation breakpoints in 1q44 implicates the serine/threonine kinase AKT3 in postnatal microcephaly and agenesis of the corpus callosum. Am. J. Hum. Genet. 81, 292-303 (2007).

38. Wang, D., Zeesman, S., Tarnopolsky, M.A. \& Nowaczyk, M.J. Duplication of AKT3 as a cause of macrocephaly in duplication 1q43q44. Am. J. Med. Genet. A. 161A, 2016-2019 (2013).
39. Pawlikowska, L. et al. Association of common genetic variation in the insulin/IGF1 signaling pathway with human longevity. Aging Cell 8, 460-472 (2009).

40. Davies, G. et al. Genetic contributions to variation in general cognitive function: a meta-analysis of genome-wide association studies in the CHARGE consortium ( $N=53,949)$. Mol. Psychiatry 20, 183-192 (2015).

41. de Moor, M.H.M. et al. Meta-analysis of genome-wide association studies for neuroticism, and the polygenic association with major depressive disorder. JAMA Psychiatry 72, 642-650 (2015)

42. van den Berg, S.M. et al. Meta-analysis of genome-wide association studies for extraversion: findings from the Genetics of Personality Consortium. Behav. Genet. 46, 170-182 (2016)

43. Nalls, M.A. et al. Large-scale meta-analysis of genome-wide association data identifies six new risk loci for Parkinson's disease. Nat. Genet. 46, 989-993 (2014).

44. Cross-Disorder Group of the Psychiatric Genomics Consortium. Identification of risk loci with shared effects on five major psychiatric disorders: a genome-wide analysis. Lancet 381, 1371-1379 (2013).

45. Benyamin, B. et al. Childhood intelligence is heritable, highly polygenic and associated with FNBP1L. Mol. Psychiatry 19, 253-258 (2014).

46. Kundaje, A. et al. Integrative analysis of 111 reference human epigenomes. Nature 518, 317-330 (2015).

Hieab H H Adams ${ }^{1,2,261}$, Derrek P Hibar ${ }^{3,261}$, Vincent Chouraki ${ }^{4-6,261}$, Jason L Stein ${ }^{3,7,261}$, Paul A Nyquist ${ }^{8,261}$, Miguel E Rentería ${ }^{9,261}$, Stella Trompet ${ }^{10,261}$, Alejandro Arias-Vasquez ${ }^{11-14,261}$, Sudha Seshadri ${ }^{4,6}$, Sylvane Desrivières ${ }^{15}$, Ashley H Beecham ${ }^{16,17}$, Neda Jahanshad ${ }^{3}$, Katharina Wittfeld ${ }^{18,19}$, Sven J Van der Lee ${ }^{1}$, Lucija Abramovic ${ }^{20}$, Saud Alhusaini' ${ }^{21,22}$, Najaf Amin ${ }^{1}$, Micael Andersson ${ }^{23}$, Konstantinos Arfanakis ${ }^{24-26}$, Benjamin S Aribisala ${ }^{27-29}$, Nicola J Armstrong ${ }^{30,31}$, Lavinia Athanasiu ${ }^{32,33}$, Tomas Axelsson ${ }^{34}$, Alexa Beiser ${ }^{4,6,35}$, Manon Bernard ${ }^{36}$, Joshua C Bis ${ }^{37}$, Laura M E Blanken ${ }^{38,39}$, Susan H Blanton ${ }^{16,17}$, Marc M Bohlken ${ }^{20}$, Marco P Boks ${ }^{20}$, Janita Bralten ${ }^{11,14}$, Adam M Brickman ${ }^{40-42}$, Owen Carmichael ${ }^{43}$, M Mallar Chakravarty ${ }^{44,45}$, Ganesh Chauhan $^{46}$, Qiang Chen ${ }^{47}$, Christopher R K Ching ${ }^{3,48}$, Gabriel Cuellar-Partida ${ }^{9}$, Anouk Den Braber ${ }^{49}$, Nhat Trung Doan ${ }^{32}$, Stefan Ehrlich ${ }^{50-52}$, Irina Filippi ${ }^{53,54}$, Tian Ge $\mathrm{G}^{52,55-57}$, Sudheer Giddaluru ${ }^{58,59}$, Aaron L Goldman ${ }^{47}$, Rebecca F Gottesman ${ }^{60}$, Corina U Greven ${ }^{13,61,62}$, Oliver Grimm ${ }^{63}$, Michael E Griswold ${ }^{64}$, Tulio Guadalupe ${ }^{65,66}$, Johanna Hass ${ }^{67}$, Unn K Haukvik ${ }^{32,68}$, Saima Hilal ${ }^{69,70}$, Edith Hofer ${ }^{71,72}$, David Hoehn ${ }^{73}$, Avram J Holmes ${ }^{51,74}$, Martine Hoogman ${ }^{11,14}$, Deborah Janowitz ${ }^{19}$, Tianye Jia ${ }^{15}$, Dalia Kasperaviciute ${ }^{75,76}$, Sungeun Kim ${ }^{77-79}$, Marieke Klein ${ }^{11,14}$, Bernd Kraemer ${ }^{80}$, Phil H Lee ${ }^{51,55-57,81}$, Jiemin Liao ${ }^{82}$, David C M Liewald ${ }^{83}$, Lorna M Lopez ${ }^{83}$, Michelle Luciano ${ }^{83}$, Christine Macare ${ }^{15}$, Andre Marquand ${ }^{14,84}$, Mar Matarin ${ }^{75,85}$, Karen A Mather ${ }^{30}$, Manuel Mattheisen ${ }^{86-88}$, Bernard Mazoyer ${ }^{89}$, David R McKay ${ }^{90,91}$, Rebekah McWhirter ${ }^{92}$, Yuri Milaneschi ${ }^{93}$, Nazanin Mirza-Schreiber ${ }^{73}$, Ryan L Muetzel ${ }^{38,39}$, Susana Muñoz Maniega ${ }^{27,29,83}$, Kwangsik Nho ${ }^{77-79}$, Allison C Nugent ${ }^{94}$, Loes M Olde Loohuis ${ }^{95}$, Jaap Oosterlaan ${ }^{96}$, Martina Papmeyer ${ }^{97,98}$, Irene Pappa $^{38,99}$, Lukas Pirpamer ${ }^{71}$, Sara Pudas ${ }^{23}$, Benno Pütz ${ }^{73}$, Kumar B Rajan ${ }^{100}$, Adaikalavan Ramasamy ${ }^{85,101,102}$, Jennifer S Richards ${ }^{13,14,61}$, Shannon L Risacher ${ }^{77,79}$, Roberto Roiz-Santiañez ${ }^{103,104}$, Nanda Rommelse ${ }^{12,14,61}$, Emma J Rose ${ }^{105}$, Natalie A Royle ${ }^{27,29,83,106}$, Tatjana Rundek 107,108, Philipp G Sämann ${ }^{73}$, Claudia L Satizabal ${ }^{4,6}$, Lianne Schmaal ${ }^{109-111}$, Andrew J Schork ${ }^{112,113}$, Li Shen ${ }^{77-79}$, Jean Shin ${ }^{36}$, Elena Shumskaya ${ }^{11,14,84}$, Albert V Smith ${ }^{114,115}$, Emma Sprooten ${ }^{90,91,97,116}$, Lachlan T Strike ${ }^{9,117}$, Alexander Teumer ${ }^{118}$, Russell Thomson ${ }^{119}$, Diana Tordesillas-Gutierrez ${ }^{104,120}$, Roberto Toro ${ }^{121}$, Daniah Trabzuni ${ }^{85,122}$, Dhananjay Vaidya ${ }^{123}$, Jeroen Van der Grond ${ }^{124}$, Dennis Van der Meer ${ }^{125}$, Marjolein M J Van Donkelaar ${ }^{11,14}$, Kristel R Van Eijk ${ }^{126}$, Theo G M Van Erp ${ }^{127}$, Daan Van Rooij ${ }^{13,14,125}$, Esther Walton ${ }^{67}$, Lars T Westlye ${ }^{33,128}$, Christopher D Whelan ${ }^{3,22}$, Beverly G Windham ${ }^{129}$, Anderson M Winkler ${ }^{90,130}$, Girma Woldehawariat ${ }^{94}$, Christiane Wolf ${ }^{131}$, Thomas Wolfers ${ }^{11,14}$, Bing Xu ${ }^{15}$, Lisa R Yanek ${ }^{123}$, Jingyun Yang ${ }^{25,132}$, Alex Zijdenbos ${ }^{133}$, Marcel P Zwiers ${ }^{14,84}$, Ingrid Agartz $^{32,68,134}$, Neelum T Aggarwal ${ }^{25,100,132}$, Laura Almasy ${ }^{135-137}$, David Ames ${ }^{138,139}$, Philippe Amouyel ${ }^{5}$, Ole A Andreassen ${ }^{32,33}$, Sampath Arepalli ${ }^{140}$, Amelia A Assareh ${ }^{30}$, Sandra Barral ${ }^{40}$, Mark E Bastin ${ }^{27,29,83,106,}$ Diane M Becker ${ }^{123}$, James T Becker ${ }^{141}$, David A Bennett ${ }^{25,132}$, John Blangero ${ }^{135}$, Hans van Bokhoven ${ }^{11,14}$, Dorret I Boomsma ${ }^{49}$, Henry Brodaty ${ }^{30,142}$, Rachel M Brouwer ${ }^{20}$, Han G Brunner ${ }^{11,14,143}$, Randy L Buckner ${ }^{51,144}$, Jan K Buitelaar ${ }^{13,14,61}$, Kazima B Bulayeva ${ }^{145}$, Wiepke Cahn ${ }^{20}$, Vince D Calhoun ${ }^{146,147}$, Dara M Cannon ${ }^{94,148,}$ Gianpiero L Cavalleri' ${ }^{22}$, Christopher Chen ${ }^{69,70}$, Ching-Yu Cheng ${ }^{82,149,150}$, Sven Cichon ${ }^{151-153}$, Mark R Cookson ${ }^{140}$, Aiden Corvin ${ }^{105}$, Benedicto Crespo-Facorro ${ }^{103,104}$, Joanne E Curran ${ }^{135}$, Michael Czisch ${ }^{73}$, Anders M Dale ${ }^{154-158}$, Gareth E Davies ${ }^{159-162}$, Eco J C De Geus ${ }^{49}$, Philip L De Jager ${ }^{56,162,163,}$ 
Greig I de Zubicaray ${ }^{164}$, Norman Delanty 22,165 , Chantal Depondt ${ }^{166}$, Anita L DeStefano ${ }^{6,59}$, Allissa Dillman ${ }^{140}$, Srdjan Djurovic ${ }^{58,167}$, Gary Donohoe ${ }^{168,169}$, Wayne C Drevets ${ }^{94,170}$, Ravi Duggirala ${ }^{135}$, Thomas D Dyer ${ }^{135}$, Susanne Erk ${ }^{171}$, Thomas Espeseth ${ }^{33,128}$, Denis A Evans ${ }^{100}$, Iryna O Fedko ${ }^{49}$, Guillén Fernández ${ }^{13,14}$, Luigi Ferrucci $^{172}$, Simon E Fisher ${ }^{14,65}$, Debra A Fleischman ${ }^{25,173,174}$, Ian Ford ${ }^{175}$, Tatiana M Foroud ${ }^{78,176}$, Peter T Fox ${ }^{177}$, Clyde Francks ${ }^{14,65}$, Masaki Fukunaga ${ }^{178}$, J Raphael Gibbs ${ }^{85,140}$, David C Glahn ${ }^{90,91}$, Randy L Gollub ${ }^{51,52,56}$, Harald H H Göring ${ }^{135}$, Hans J Grabe ${ }^{19}$, Robert C Green ${ }^{56,179}$, Oliver Gruber ${ }^{80}$, Vilmundur Gudnason ${ }^{114,115}$, Sebastian Guelfi ${ }^{85}$, Narelle K Hansell ${ }^{9,117}$, John Hardy ${ }^{85}$, Catharina A Hartman ${ }^{125}$, Ryota Hashimoto ${ }^{180,181}$, Katrin Hegenscheid ${ }^{182}$, Andreas Heinz ${ }^{171}$, Stephanie Le Hellard ${ }^{58,59}$, Dena G Hernandez ${ }^{85,140,183}$, Dirk J Heslenfeld ${ }^{184}$, Beng-Choon Ho ${ }^{185}$, Pieter J Hoekstra ${ }^{125}$, Wolfgang Hoffmann ${ }^{18,118}$, Albert Hofman $^{1}$, Florian Holsboer ${ }^{73,186}$, Georg Homuth ${ }^{187}$, Norbert Hosten ${ }^{182}$, Jouke-Jan Hottenga ${ }^{49}$, Hilleke E Hulshoff Pol $^{20}$, Masashi Ikeda ${ }^{188}$, M Kamran Ikram ${ }^{1,69,70,82,149}$, Clifford R Jack Jr ${ }^{189}$, Mark

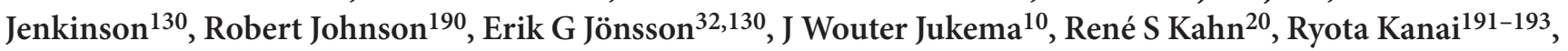
Iwona Kloszewska ${ }^{194}$, David S Knopman ${ }^{195}$, Peter Kochunov ${ }^{196}$, John B Kwok ${ }^{197,198}$, Stephen M Lawrie ${ }^{97}$, Hervé Lemaître ${ }^{53,54}$, Xinmin Liu ${ }^{94,199}$, Dan L Longo ${ }^{200}$, W T Longstreth Jr201,202, Oscar L Lopez ${ }^{203,204}$, Simon Lovestone ${ }^{205,206}$, Oliver Martinez ${ }^{207}$, Jean-Luc Martinot ${ }^{53,54}$, Venkata S Mattay 47,60,208, Colm McDonald ${ }^{148}$, Andrew M McIntosh ${ }^{83,97}$, Katie L McMahon ${ }^{209}$, Francis J McMahon ${ }^{94}$, Patrizia Mecocci ${ }^{210}$, Ingrid Melle ${ }^{32,33}$, Andreas Meyer-Lindenberg ${ }^{63}$, Sebastian Mohnke ${ }^{171}$, Grant W Montgomery ${ }^{9}$, Derek W Morris ${ }^{168,169}$, Thomas H Mosley ${ }^{129}$, Thomas W Mühleisen ${ }^{148,153}$, Bertram Müller-Myhsok ${ }^{73,211,212}$, Michael A Nalls ${ }^{140}$, Matthias Nauck ${ }^{213,214}$, Thomas E Nichols ${ }^{130,215}$, Wiro J Niessen ${ }^{2,216,217}$, Markus M Nöthen ${ }^{152,218}$, Lars Nyberg ${ }^{23}$, Kazutaka Ohi ${ }^{180}$, Rene L Olvera ${ }^{177}$, Roel A Ophoff ${ }^{20,95}$, Massimo Pandolfo ${ }^{166}$, Tomas Paus ${ }^{219-221}$, Zdenka Pausova ${ }^{36,222,223}$, Brenda W J H Penninx ${ }^{111}$, G Bruce Pike ${ }^{224,225}$, Steven G Potkin ${ }^{127}$, Bruce M Psaty ${ }^{226-229}$, Simone Reppermund ${ }^{30,230}$, Marcella Rietschel ${ }^{63}$, Joshua L Roffman ${ }^{51}$, Nina Romanczuk-Seiferth ${ }^{171}$, Jerome I Rotter ${ }^{231}$, Mina Ryten ${ }^{85,101}$, Ralph L Sacco ${ }^{17,107,108,232}$, Perminder S Sachdev ${ }^{30,233}$, Andrew J Saykin ${ }^{77,79,176,}$ Reinhold Schmidt ${ }^{71}$, Peter R Schofield ${ }^{197,198}$, Sigurdur Sigurdsson ${ }^{114}$, Andy Simmons ${ }^{234-236}$, Andrew Singleton ${ }^{140}$, Sanjay M Sisodiya ${ }^{75}$, Colin Smith ${ }^{237}$, Jordan W Smoller ${ }^{51,55-57}$, Hilkka Soininen ${ }^{238,239}$, Velandai Srikanth ${ }^{240}$, Vidar M Steen ${ }^{58,59}$, David J Stott ${ }^{241}$, Jessika E Sussmann ${ }^{97}$, Anbupalam Thalamuthu ${ }^{30}$, Henning Tiemeier ${ }^{1,39}$, Arthur W Toga ${ }^{242}$, Bryan J Traynor ${ }^{140}$, Juan Troncoso ${ }^{243}$, Jessica A Turner ${ }^{244}$, Christophe Tzourio ${ }^{245}$, Andre G Uitterlinden 1,246, Maria C Valdés Hernández 27,29,83,106, Marcel Van der Brug ${ }^{247}$, Aad Van der Lugt ${ }^{2}$, Nic J A Van der Wee ${ }^{248}$, Cornelia M Van Duijn ${ }^{1}$, Neeltje E M Van Haren ${ }^{20}$, Dennis Van 't Ent ${ }^{49}$, Marie-Jose Van Tol ${ }^{249}$, Badri N Vardarajan ${ }^{40}$, Dick J Veltman ${ }^{111}$, Meike W Vernooij ${ }^{1,2}$, Henry Völzke ${ }^{118}$, Henrik Walter ${ }^{171}$, Joanna M Wardlaw 27,29,83,106, Thomas H Wassink ${ }^{250}$, Michael E Weale ${ }^{101}$, Daniel R Weinberger ${ }^{47,251}$, Michael W Weiner ${ }^{252}$, Wei Wen ${ }^{30}$, Eric Westman ${ }^{253}$, Tonya White ${ }^{2,39}$, Tien Y Wong ${ }^{82,145,150}$, Clinton B Wright ${ }^{107,108,232}$, H Ronald Zielke ${ }^{190}$, Alan B Zonderman ${ }^{254}$, the Alzheimer's Disease Neuroimaging Initiative, EPIGEN, IMAGEN, SYS, Ian J Deary ${ }^{83}$, Charles DeCarli ${ }^{207}$, Helena Schmidt ${ }^{255}$, Nicholas G Martin ${ }^{9}$, Anton J M De Craen ${ }^{256}$, Margaret J Wright ${ }^{117,209,262}$, Lenore J Launer ${ }^{257,262}$, Gunter Schumann ${ }^{15,262}$, Myriam Fornage ${ }^{258,262}$, Barbara Franke ${ }^{11,12,14,262}$, Stéphanie Debette $e^{4,47,259,262}$, Sarah E Medland ${ }^{9,262}$, M Arfan Ikram 1,2,260,262 \& Paul M Thompson ${ }^{3,262}$

\footnotetext{
${ }^{1}$ Department of Epidemiology, Erasmus MC, Rotterdam, the Netherlands. ${ }^{2}$ Department of Radiology and Nuclear Medicine, Erasmus MC, Rotterdam, the Netherlands. ${ }^{3}$ Imaging Genetics Center, USC Mark and Mary Stevens Neuroimaging and Informatics Institute, Keck School of Medicine of University of Southern California, Los Angeles, California, USA. ${ }^{4}$ Department of Neurology, Boston University School of Medicine, Boston, Massachusetts, USA. ${ }^{5}$ ille University, Inserm, CHU Lille, Institut Pasteur de Lille, U1167 - RID-AGE - Risk factors and molecular determinants of aging-related diseases, Lille, France. ${ }^{6}$ Framingham Heart Study, Framingham, Massachusetts, USA. 7 Department of Genetics and UNC Neuroscience Center, University of North Carolina (UNC), Chapel Hill, North Carolina, USA. ${ }^{8}$ Department of Neurology, Department of Anesthesia/Critical Care Medicine, Department of Neurosurgery, Johns Hopkins University, Baltimore, Maryland, USA. ${ }^{9}$ QIMR Berghofer Medical Research Institute, Brisbane, Australia. ${ }^{10}$ Department of Cardiology, Leiden University Medical Center, Leiden, the Netherlands.

${ }^{11}$ Department of Human Genetics, Radboud University Medical Center, Nijmegen, the Netherlands. ${ }^{12}$ Department of Psychiatry, Radboud University Medical Center, Nijmegen, the Netherlands. ${ }^{13}$ Department of Cognitive Neuroscience, Radboud University Medical Center, Nijmegen, the Netherlands. ${ }^{14}$ Donders Institute for Brain, Cognition and Behaviour, Radboud University, Nijmegen, the Netherlands. ${ }^{15}$ MRC-SGDP Centre, Institute of Psychiatry, Psychology and Neuroscience, King's College London, London, UK. ${ }^{16}$ Dr. John T. Macdonald Foundation Department of Human Genetics, University of Miami, Miller School of Medicine, Miami, Florida, USA. ${ }^{17} \mathrm{~J}$ John P. Hussman Institute for Human Genomics, University of Miami, Miller School of Medicine, Miami, Florida, USA. ${ }^{18}$ German Center for Neurodegenerative Diseases (DZNE) Rostock/Greifswald, Greifswald, Germany. ${ }^{19}$ Department of Psychiatry, University Medicine Greifswald, Greifswald, Germany. ${ }^{20}$ Brain Center Rudolf Magnus, Department of Psychiatry, UMC Utrecht, Utrecht, the Netherlands. ${ }^{21}$ Department of Neurology and Neurosurgery, Montreal Neurological Institute, McGill University, Montreal, Canada. 22The Royal College of Surgeons in Ireland, Dublin 2, Ireland. ${ }^{23}$ Department of Integrative Medical Biology and Umeå center for Functional Brain Imaging, Umeå University, Umeå, Sweden. ${ }^{24}$ Department of Biomedical Engineering, Illinois Institute of Technology, Chicago, Illinois, USA. 25Rush Alzheimer's Disease Center, Rush University Medical Center, Chicago, Illinois, USA. 26Department of Diagnostic Radiology and Nuclear Medicine, Rush University Medical Center, Chicago, Illinois, USA. ${ }^{27}$ Brain Research Imaging Centre, University of Edinburgh, Edinburgh, UK. ${ }^{28}$ Department of Computer Science,
} 
Lagos State University, Lagos, Nigeria. ${ }^{29}$ Scottish Imaging Network, A Platform for Scientific Excellence (SINAPSE) Collaboration, Department of Neuroimaging Sciences, University of Edinburgh, Edinburgh, UK. ${ }^{30}$ Centre for Healthy Brain Ageing, School of Psychiatry, University of New South Wales, Sydney, Australia. ${ }^{31}$ Mathematics and Statistics, Murdoch University, Perth, Australia. ${ }^{32}$ NORMENT - KG Jebsen Centre, Institute of Clinical Medicine, University of Oslo, Oslo, Norway. ${ }^{33}$ NORMENT - KG Jebsen Centre, Division of Mental Health and Addiction, Oslo University Hospital, Oslo, Norway. ${ }^{34}$ Department of Medical Sciences, Molecular Medicine and Science for Life Laboratory, Uppsala University, Uppsala, Sweden. ${ }^{35}$ Department of Biostatistics, Boston University School of Public Health, Boston, Massachusetts, USA. ${ }^{36}$ Hospital for Sick Children, University of Toronto, Toronto, Canada. ${ }^{37}$ Cardiovascular Health Research Unit, Department of Medicine, University of Washington, Seattle, Washington, USA. ${ }^{38}$ Generation R Study Group, Erasmus Medical Center, Rotterdam, the Netherlands. ${ }^{39}$ Department of Child and Adolescent Psychiatry/Psychology, Erasmus MC-Sophia Children's Hospital, Rotterdam, the Netherlands. ${ }^{40}$ Taub Institute for Research on Alzheimer's Disease and the Aging Brain, Columbia University Medical Center, New York, New York, USA. ${ }^{11}$ G. H. Sergievsky Center, Columbia University Medical Center, New York, New York, USA. ${ }^{42}$ Department of Neurology, Columbia University Medical Center, New York, New York, USA. ${ }^{33}$ Pennington Biomedical Research Center, Baton Rouge, Louisiana, USA. ${ }^{44}$ Cerebral Imaging Centre, Douglas Mental Health University Institute, Montreal, Canada. ${ }^{45}$ Department of Psychiatry and Biomedical Engineering, McGill University, Montreal, Canada. ${ }^{46}$ INSERM Unit U1219, University of Bordeaux, France. ${ }^{47}$ Lieber Institute for Brain Development, Baltimore, Maryland, USA. 48Interdepartmental Neuroscience Graduate Program, UCLA School of Medicine, Los Angeles, California, USA. ${ }^{49}$ Biological Psychology, Neuroscience Campus Amsterdam, Vrije Universiteit University and Vrije Universiteit Medical Center, Amsterdam, the Netherlands. ${ }^{50}$ Division of Psychological and Social Medicine and Developmental Neurosciences, Faculty of Medicine, TU Dresden, Germany. ${ }^{51}$ Department of Psychiatry, Massachusetts General Hospital, Boston, Masschusetts, USA.

${ }^{52}$ Martinos Center for Biomedical Imaging, Massachusetts General Hospital, Charlestown, North Carolina, USA. ${ }^{53}$ NSERM Unit 1000 "Neuroimaging and Psychiatry", University Paris Sud, University Paris Descartes, Paris, France. ${ }^{54}$ Maison de Solenn, Adolescent Psychopathology and Medicine Department, APHP Hospital Cochin, Paris, France. ${ }^{55}$ Psychiatric and Neurodevelopmental Genetics Unit, Center for Human Genetic Research, Massachusetts General Hospital, Boston, Massachusetts, USA. ${ }^{56}$ Harvard Medical School, Boston, Massachusetts, USA. ${ }^{57}$ Stanley Center for Psychiatric Research, Broad Institute of MIT and Harvard, Boston, Massachusetts, USA. ${ }^{58}$ NORMENT - KG Jebsen Centre for Psychosis Research, Department of Clinical Science, University of Bergen, Norway. ${ }^{59}$ Dr. Einar Martens Research Group for Biological Psychiatry, Center for Medical Genetics and Molecular Medicine, Haukeland University Hospital, Bergen, Norway. ${ }^{60}$ Department of Neurology, Johns Hopkins University School of Medicine, Baltimore, Maryland, USA. ${ }^{61}$ Karakter Child and Adolescent Psychiatry University Center, Nijmegen, the Netherlands. 62 King's College London, Medical Research Council Social, Genetic and Developmental Psychiatry Centre, Institute of Psychology, Psychiatry and Neurosciene, London, UK. ${ }^{63}$ Central Institute of Mental Health, Medical Faculty Mannheim, University Heidelberg, Mannheim, Germany. ${ }^{64}$ Center of Biostatistics and Bioinformatics, University of Mississippi Medical Center, Jackson, Mississippi, USA. ${ }^{65}$ Language and Genetics Department, Max Planck Institute for Psycholinguistics, Nijmegen, the Netherlands. ${ }^{66}$ International Max Planck Research School for Language Sciences, Nijmegen, the Netherlands. ${ }^{67}$ Department of Child and Adolescent Psychiatry, Faculty of Medicine of the TU Dresden, Dresden, Germany. ${ }^{68}$ Department of Research and Development, Diakonhjemmet Hospital, Oslo, Norway. ${ }^{69}$ Department of Pharmacology, National University of Singapore, Singapore. ${ }^{70}$ Memory Aging and Cognition Centre (MACC), National University Health System, Singapore. ${ }^{71}$ Department of Neurology, Clinical Division of Neurogeriatrics, Medical University Graz, Austria, Graz, Austria. ${ }^{72}$ Institute of Medical Informatics, Statistics and Documentation, Medical University Graz, Austria, Graz, Austria. ${ }^{73}$ Max Planck Institute of Psychiatry, Department of Translational Research in Psychiatry, Munich, Germany. ${ }^{74}$ Department of Psychology, Yale University, New Haven, Connecticut, USA. 75 UCL Institute of Neurology, London, United Kingdom and Epilepsy Society, Bucks, UK. ${ }^{76}$ Department of Medicine, Imperial College London, London, UK. ${ }^{77}$ Center for Neuroimaging, Radiology and Imaging Sciences, Indiana University School of Medicine, Indianapolis, Indiana, USA. ${ }^{78}$ Center for Computational Biology and Bioinformatics, Indiana University School of Medicine, Indianapolis, Indiana, USA. ${ }^{79}$ Indiana Alzheimer Disease Center, Indiana University School of Medicine, Indianapolis, Indiana, USA. ${ }^{80}$ Section for Experimental Psychopathology and Neuroimaging, Department of General Psychiatry, Heidelberg University, Heidelberg, Germany. ${ }^{81}$ Lurie Center for Autism, Massachusetts General Hospital, Harvard Medical School, Lexington, Massachusetts, USA. ${ }^{82}$ Singapore Eye Research Institute, Singapore National Eye Centre, Singapore. ${ }^{83}$ Centre for Cognitive Ageing and Cognitive Epidemiology, Psychology, University of Edinburgh, Edinburgh, UK. ${ }^{84}$ Donders Centre for Cognitive Neuroimaging, Radboud University, Nijmegen, The Netherlands. ${ }^{85}$ Reta Lila Weston Institute and Department of Molecular Neuroscience, UCL Institute of Neurology, London, UK. ${ }^{86}$ Department of Biomedicine, Aarhus University, Aarhus, Denmark. ${ }^{87}$ The Lundbeck Foundation Initiative for Integrative Psychiatric Research, iPSYCH, Aarhus and Copenhagen, Denmark. ${ }^{88}$ Center for integrated Sequencing, iSEQ, Aarhus University, Aarhus, Denmark. ${ }^{89}$ UMR5296 University of Bordeaux, CNRS, CEA, Bordeaux, France.

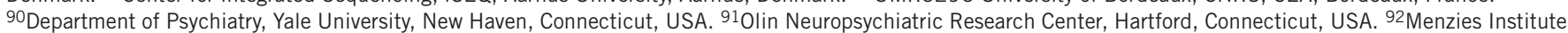
for Medical Research, University of Tasmania, Hobart, Tasmania, Australia. ${ }^{93}$ Department of Psychiatry, EMGO Institute for Health and Care Research and Neuroscience Campus Amsterdam, VU University Medical Center/GGZ inGeest, Amsterdam, The Netherlands. ${ }^{94}$ Experimental Therapeutics and Pathophysiology Branch, National Institute of Mental Health Intramural Research Program, National Institutes of Health, Bethesda, Maryland, USA. ${ }^{55}$ Center for Neurobehavioral Genetics, University of California, Los Angeles, California, USA. ${ }^{66}$ Department of Clinical Neuropsychology, VU University Amsterdam, Amsterdam, the Netherlands. 97Division of Psychiatry, Royal Edinburgh Hospital, University of Edinburgh, Edinburgh, UK. 98Division of Systems Neuroscience of Psychopathology, Translational Research Center, University Hospital of Psychiatry, University of Bern, Switzerland. ${ }^{99}$ School of Pedagogical and Educational Sciences, Erasmus University Rotterdam, Rotterdam, the Netherlands. ${ }^{100}$ Rush Institute for Healthy Aging, Rush University Medical Center, Chicago, Illinois, USA. 101Department of Medical and Molecular Genetics, King's College London, London, UK. 102The Jenner Institute Laboratories, University of Oxford, Oxford, UK. 103Department of Medicine and Psychiatry, University Hospital Marqués de Valdecilla, School of Medicine, University of Cantabria-IDIVAL, Santander, Spain. ${ }^{104}$ CIBERSAM (Centro Investigación Biomédica en Red Salud Mental), Santander, Spain. ${ }^{105}$ Psychosis Research Group, Department of Psychiatry and Trinity Translational Medicine Institute, Trinity College Dublin. ${ }^{106}$ Centre for Clinical Brain Sciences, University of Edinburgh, Edinburgh, UK. ${ }^{107}$ Department of Neurology, University of Miami, Miller School of Medicine, Miami, Florida, USA. ${ }^{108}$ Department of Epidemiology and Public Health Sciences, University of Miami, Miller School of Medicine, Miami, Florida, USA. ${ }^{109}$ Orygen, The National Centre of Excellence in Youth Mental Health, Melbourne, VIC, Australia. ${ }^{110}$ Centre for Youth Mental Health, The University of Melbourne, Melbourne, VIC, Australia. ${ }^{111}$ Department of Psychiatry, Neuroscience Campus Amsterdam, VU University Medical Center, Amsterdam, the Netherlands. 112 Multimodal Imaging Laboratory, Department of Neurosciences, University of California, San Diego, USA. ${ }^{113}$ Department of Cognitive Sciences, University of California, San Diego, USA. ${ }^{114}$ Icelandic Heart Association, Kopavogur, Iceland. ${ }^{115}$ Faculty of Medicine, University of Iceland, Reykjavik, Iceland. ${ }^{116}$ Department of Psychiatry, Icahn School of Medicine at Mount Sinai, New York, New York, USA. ${ }^{117}$ Queensland Brain Institute, University of Queensland, Brisbane, Australia. ${ }^{118}$ Institute for Community Medicine, University Medicine Greifswald, Greifswald, Germany. ${ }^{119}$ School of Computing Engineering and Mathematics, Western Sydney University, Parramatta, Australia. ${ }^{120}$ Neuroimaging Unit, Technological Facilities. Valdecilla Biomedical Research Institute IDIVAL, Santander, Cantabria, Spain. 121Institut Pasteur, Paris, France. ${ }^{122}$ Department of Genetics, King Faisal Specialist Hospital and Research Centre, Riyadh, Saudi Arabia. ${ }^{123}$ GeneSTAR Research Center, Department of Medicine, Johns Hopkins University School of Medicine, Baltimore, Maryland, USA. ${ }^{124}$ Department of Radiology, Leiden University Medical Center, Leiden, the Netherlands. ${ }^{125}$ Department of Psychiatry, University of Groningen, University Medical Center Groningen, Groningen, the Netherlands. ${ }^{126}$ Brain Center Rudolf Magnus, Human Neurogenetics Unit, UMC Utrecht, Utrecht, the Netherlands. ${ }^{127}$ Department of Psychiatry and Human Behavior, University of California-Irvine, Irvine, California, USA. ${ }^{128}$ NORMENT - KG Jebsen Centre, Department of Psychology, University of Oslo, Oslo, Norway. ${ }^{129}$ Department of Medicine, University of Mississippi

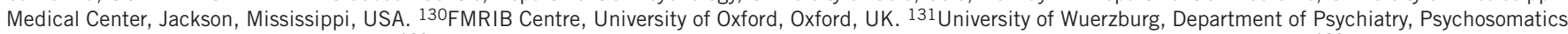
and Psychotherapy, Wuerzburg, Germany. ${ }^{132}$ Department of Neurological Sciences, Rush University Medical Center, Chicago, Illinois, USA. ${ }^{133}$ Biospective Inc, Montreal, Quebec, Canada, Montréal, Québec, Canada. ${ }^{134}$ Department of Clinical Neuroscience, Centre for Psychiatric Research, Karolinska Institutet, Stockholm, Sweden. ${ }^{135}$ South Texas Diabetes and Obesity Institute, University of Texas Rio Grande Valley School of Medicine Brownsville/Edinburg/San Antonio, Texas, USA. ${ }^{136}$ Department of Genetics, Perelman School of Medicine, University of Pennsylvania, Philadelphia, Pennsylvania, USA. ${ }^{137}$ Department of Biomedical and Health Informatics, The Children's Hospital of Philadelphia, Philadelphia, Pennsylvania, USA. ${ }^{138}$ National Ageing Research Institute, Royal Melbourne Hospital, Melbourne, Australia. ${ }^{139}$ Academic Unit for Psychiatry of Old Age, University of Melbourne, Melbourne, Australia. ${ }^{140}$ Laboratory of Neurogenetics, National Institute on Aging, National Institutes of Health, Bethesda, Maryland, USA. ${ }^{141}$ Departments of Psychiatry, Neurology, and Psychology, University of Pittsburgh, Pittsburgh, Pennsylvania, USA. ${ }^{142}$ Dementia Collaborative Research Centre - Assessment and Better Care, UNSW, Sydney, Australia. ${ }^{143}$ Department of Clinical Genetics, Maastricht University Medical Center, Maastricht, the Netherlands. ${ }^{144}$ Department of Psychology, Center for Brain Science, Harvard University, Cambridge, Massachusetts, USA. ${ }^{145}$ Department of Evolution and Genetics, Dagestan State University, Makhachkala, Dagestan, Russia. ${ }^{146}$ The Mind Research Network and LBERI, Albuquerque, New Mexico, USA. ${ }^{147}$ Department of ECE, University of New Mexico, Albuquerque, New Mexico, USA. ${ }^{148}$ Centre for Neuroimaging and Cognitive Genomics (NICOG), Clinical Neuroimaging Laboratory, NCBES Galway Neuroscience Centre, College of Medicine Nursing and Health Sciences, National University of Ireland Galway, Galway, Ireland. ${ }^{149}$ Academic Medicine Research Institute, Duke-NUS Graduate Medical School, Singapore. ${ }^{150}$ Department of Ophthalmology, Yong Loo Lin School of Medicine, National University of Singapore, Singapore. ${ }^{151}$ Division of Medical Genetics, Department of Biomedicine, University of Basel, Basel, Switzerland. 
152 Institute of Human Genetics, University of Bonn, Bonn, Germany. ${ }^{153}$ Institute of Neuroscience and Medicine (INM-1), Research Centre Jülich, Jülich, Germany. ${ }^{154}$ Center for Multimodal Imaging and Genetics, University of California, San Diego, California, USA. ${ }^{155}$ Department of Neurosciences, University of California, San Diego, California, USA. ${ }^{156}$ Department of Radiology, University of California, San Diego, California, USA. ${ }^{157}$ Department of Psychiatry, University of California, San Diego, California, USA. ${ }^{158}$ Department of Cognitive Science, University of California, San Diego, California, USA. ${ }^{159}$ Avera Institute for Human Genetics, Sioux Falls, South Dakota, USA. 160Program in Translational NeuroPsychiatric Genomics, Departments of Neurology and Psychiatry, Brigham and Women's Hospital, Boston, Massachusetts, USA. ${ }^{161}$ Harvard Medical School, Boston, Massachusetts, USA. 162Program in Medical and Population Genetics, Broad Institute, Cambridge, Massachusetts, USA. ${ }^{163}$ Broad Institute, Cambridge, Massachusetts, USA. ${ }^{164}$ Faculty of Health and Institute of Health and Biomedical Innovation, Queensland University of Technology (QUT), Brisbane, Australia. ${ }^{165}$ Neurology Division, Beaumont Hospital, Dublin, 9, Ireland. ${ }^{166}$ Department of Neurology, Hopital Erasme, Universite Libre de Bruxelles, Brussels, Belgium. ${ }^{167}$ Department of Medical Genetics, Oslo University Hospital, Oslo, Norway. ${ }^{168}$ Cognitive Genetics and Cognitive Therapy Group, Neuroimaging, Cognition and Genomics Centre (NICOG) and NCBES Galway Neuroscience Centre, School of Psychology and Discipline of Biochemistry, National University of Ireland Galway, Galway, Ireland. ${ }^{169}$ Neuropsychiatric Genetics Research Group, Department of Psychiatry and Trinity College Institute of Psychiatry, Trinity College Dublin, Dublin 8, Ireland. 170 Janssen Research and Development, LLC, Titusville, New Jersey, USA. ${ }^{71}$ Department of Psychiatry and Psychotherapy, Charité Universitätsmedizin Berlin, CCM, Berlin, Germany. ${ }^{172}$ Intramural Research Program of the National Institute on Aging, Baltimore, Maryland, USA. ${ }^{173}$ Department of Neurological Sciences, Rush University Medical Center, Chicago, Illinois, USA. ${ }^{174}$ Department of Behavioral Sciences, Rush University Medical Center, Chicago, Illinois, USA. ${ }^{175}$ Robertson Center for Biostatistics, University of Glasgow, Glasgow, UK. ${ }^{176}$ Medical and Molecular Genetics, Indiana University School of Medicine, Indianapolis, Indiana, USA. ${ }^{177}$ University of Texas Health Science Center, San Antonio, Texas, USA. ${ }^{178}$ Division of Cerebral Integration, National Institute for Physiological Sciences, Aichi, Japan. ${ }^{179}$ Division of Genetics, Department of Medicine, Brigham and Women's Hospital, Boston, Massachusetts, USA. ${ }^{180}$ Department of Psychiatry, Osaka University Graduate School of Medicine, Osaka, Japan. ${ }^{181}$ Molecular Research Center for Children's Mental Development, United Graduate School of Child Development, Osaka University, Osaka, Japan. 182Institute of Diagnostic Radiology and Neuroradiology, University Medicine Greifswald, Greifswald, Germany. ${ }^{183}$ German Center for Neurodegenerative Diseases (DZNE), Tübingen, Germany. ${ }^{184}$ Department of Psychology, VU University Amsterdam, Amsterdam, the Netherlands. ${ }^{185}$ Department of Psychiatry, University of lowa, Iowa City, Iowa, USA. ${ }^{186} \mathrm{HMNC}$ Brain Health, Munich, Germany. ${ }^{187}$ Interfaculty Institute for Genetics and Functional Genomics, University Medicine Greifswald, Greifswald, Germany. ${ }^{188}$ Department of Psychiatry, Fujita Health University School of Medicine, Toyoake, Japan. ${ }^{189}$ Department of Radiology, Mayo Clinic, Rochester, Minnesota, USA. 190NICHD Brain and Tissue Bank for Developmental Disorders, University of Maryland Medical School, Baltimore, Maryland, USA. ${ }^{191}$ School of Psychology, University of Sussex, Brighton, UK. ${ }^{192}$ Institute of Cognitive Neuroscience, University College London, London, UK. ${ }^{193}$ Department of Neuroinformatics, Araya Brain Imaging, Tokyo, Japan. ${ }^{194}$ Medical University of Lodz, Lodz, Poland. ${ }^{195}$ Department of Neurology, Mayo Clinic, Rochester, Minnesota, USA. ${ }^{196}$ Maryland Psychiatric Research Center, Department of Psychiatry, University of Maryland School of Medicine, Baltimore, Maryland, USA. ${ }^{197}$ Neuroscience Research Australia, Sydney, Australia. ${ }^{198}$ School of Medical Sciences, UNSW, Sydney, Australia. ${ }^{199}$ Columbia University Medical Center, New York, New York, USA. ${ }^{200}$ Laboratory of Genetics, National Institute on Aging, National Institutes of Health, Baltimore, Maryland, USA. ${ }^{201}$ Department of Neurology, University of Washington, Seattle, Washington, USA. ${ }^{202}$ Department of Epidemiology, University of Washington, Seattle, Washington, USA. ${ }^{203}$ Department of Neurology, University of Pittsburgh, Pittsburgh, Pennsylvania, USA. ${ }^{204}$ Department of Psychiatry, University of Pittsburgh, Pittsburgh, Pennsylvania, USA. ${ }^{205}$ Department of Psychiatry, University of Oxford, Oxford, UK. ${ }^{206}$ NIHR Dementia Biomedical Research Unit, King's College London, London, UK. ${ }^{207}$ Imaging of Dementia and Aging (IDeA) Laboratory, Department of Neurology and Center for Neuroscience, University of California at Davis, Sacramento, California, USA. ${ }^{208}$ Department of Radiology, Johns Hopkins University School of Medicine, Baltimore, Maryland, USA. ${ }^{209}$ Centre for Advanced Imaging, University of Queensland, Brisbane, Australia. ${ }^{210}$ Section of Gerontology and Geriatrics, Department of Medicine, University of Perugia, Perugia, Italy. ${ }^{211}$ Munich Cluster for Systems Neurology (SyNergy), Munich, Germany. ${ }^{212}$ University of Liverpool, Institute of Translational Medicine, Liverpool, UK. 213 Institute of

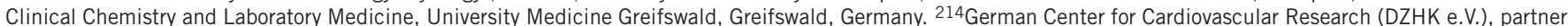
site Greifswald, Germany. ${ }^{215}$ Department of Statistics and Warwick Manufacturing Group, University of Warwick, Coventry, UK. ${ }^{216}$ Department of Medical Informatics Erasmus MC, Rotterdam, the Netherlands. ${ }^{217}$ Faculty of Applied Sciences, Delft University of Technology, Delft, the Netherlands. ${ }^{218}$ Department of Genomics, Life and Brain Center, University of Bonn, Bonn, Germany. ${ }^{219}$ Rotman Research Institute, University of Toronto, Toronto, Canada. ${ }^{220}$ Departments of Psychology and Psychiatry, University of Toronto, Canada. ${ }^{221}$ Child Mind Institute, New York, New York, USA. ${ }^{222}$ Department of Physiology, University of Toronto, Toronto, Canada. ${ }^{223}$ Department of Nutritional Sciences, University of Toronto, Toronto, Canada. ${ }^{224}$ Department of Radiology, University of Calgary, Calgary, Canada. ${ }^{225}$ Department of Clinical Neuroscience, University of Calgary, Calgary, Canada. ${ }^{226}$ Department of Epidemiology, University of Washington, Seattle, Washington, USA. ${ }^{227}$ Department of Medicine, University of Washington, Seattle, Washington, USA. ${ }^{228}$ Department of Health Services, University of Washington, Seattle, Washington, USA. ${ }^{229}$ Group Health Research Institute, Group Health, Seattle, Washington, USA. ${ }^{230}$ Department of Developmental Disability Neuropsychiatry, School of Psychiatry, UNSW Medicine, Australia. ${ }^{231}$ Institute for Translational Genomics and Population Sciences, Los Angeles Biomedical Research Institute and Pediatrics at Harbor-UCLA Medical Center, Torrance, California, USA. ${ }^{232}$ Evelyn F. McKnight Brain Institute, University of Miami, Miller School of Medicine, Miami, Florida, USA. ${ }^{233}$ Neuropsychiatric Institute, Prince of Wales Hospital, Sydney, Australia. ${ }^{234}$ Department of Neuroimaging, Institute of Psychiatry, King's College London, London, UK. ${ }^{235}$ Biomedical Research Centre for Mental Health, King's College London, London, UK. ${ }^{236}$ Biomedical Research Unit for Dementia, King's College London, London, UK. ${ }^{237}$ MRC Edinburgh Brain Bank, University of Edinburgh, Academic Department of Neuropathology, Centre for Clinical Brain Sciences, Edinburgh, UK. ${ }^{238}$ Institute of Clinical Medicine, Neurology, University of Eastern Finland, Kuopio, Finland. ${ }^{239}$ Neurocentre Neurology, Kuopio University Hospital, Finland. ${ }^{240}$ Department of Medicine, Peninsula Health and Monash University, Melbourne, Australia. ${ }^{241}$ Institute of Cardiovascular and Medical Sciences, Faculty of Medicine, University of Glasgow, Glasgow, UK. ${ }^{242}$ Laboratory of Neuro Imaging, Institute for Neuroimaging and Informatics, Keck School of Medicine of the University of Southern California, Los Angeles, California, USA. ${ }^{243}$ Brain Resource Center, Johns Hopkins University, Baltimore, Maryland, USA. ${ }^{244}$ Georgia State University, Atlanta, Georgia, USA. ${ }^{245}$ Institute for Neurodegenerative Disorders, UMR 5293, CEA, CNRS, Université de Bordeaux, France. ${ }^{246}$ Department of Internal Medicine, Erasmus MC, Rotterdam, the Netherlands. ${ }^{247}$ Genentech Inc., South San Francisco, California, USA. ${ }^{248}$ Department of Psychiatry and Leiden Institute for Brain and Cognition, Leiden University Medical Center, Leiden, The Netherlands. ${ }^{249}$ Neuroimaging Centre, University of Groningen, University Medical Center Groningen, Groningen, the Netherlands. ${ }^{250}$ Department of Psychiatry, Carver College of Medicine, University of lowa, lowa City, lowa, USA. ${ }^{251}$ Departments of Psychiatry, Neurology, Neuroscience and the Institute of Genetic Medicine, Johns Hopkins University School of Medicine, Baltimore, Maryland, USA. 252Center for Imaging of Neurodegenerative Disease, San Francisco VA Medical Center, University of California, San Francisco, California, USA. ${ }^{253}$ Department of Neurobiology, Care Sciences and Society, Karolinska Institutet, Stockholm, Sweden. ${ }^{254}$ Laboratory of Epidemiology and Population Sciences, National Institute on Aging, National Institutes of Health, Bethesda, Maryland, USA. ${ }^{255}$ Institute of Molecular Biology and Biochemistry, Medical University Graz, Graz, Austria. ${ }^{256}$ Department of Gerontology and Geriatrics, Leiden University Medical Center, Leiden, the Netherlands. ${ }^{257}$ Intramural Research Program, NIA, NIH, Bethesda, Maryland, USA. ${ }^{258}$ Institute of Molecular Medicine and Human Genetics Center, University of Texas Health Science Center at Houston, Houston, Texas, USA. ${ }^{259}$ Department of Neurology, Bordeaux University Hospital, Bordeaux, France. ${ }^{260}$ Department of Neurology, Erasmus MC, Rotterdam, the Netherlands. ${ }^{261}$ These authors contributed equally to this work. ${ }^{262}$ These authors jointly directed this work. Correspondence should be addressed to M.A.I. (m.a.ikram@erasmusmc.nl) or P.M.T. (pthomp@usc.edu). 


\section{ONLINE METHODS}

Study population. This study reports data on 32,438 subjects from 52 study sites that are part of the Cohorts for Heart and Aging Research in Genomic Epidemiology (CHARGE) $)^{10}$ consortium and Enhancing NeuroImaging Genetics through Meta-Analysis (ENIGMA) ${ }^{11}$ consortium. Briefly, the CHARGE consortium is a collaboration of predominantly population-based cohort studies that investigate the genetic and molecular underpinnings of age-related complex diseases, including those of the brain. The ENIGMA consortium brings together numerous studies, mainly with a case-control design, which performed neuroimaging in a range of neuropsychiatric or neurodegenerative diseases, as well as healthy normative populations. Studies participated in either the discovery cohort of European ancestry, the replication in European ancestry, or the generalization to other ethnicities. An overview of the demographics and type of contribution for each cohort is provided in Supplementary Table 1. Written informed consent was obtained from all participants. Each study was approved by the respective institutional review board or local ethics committee.

Genetics. Genotyping was performed using a variety of commercial arrays across the contributing sites. Both samples as well as variants underwent similar quality control procedures based on genetic homogeneity, call rate (less than $95 \%$ ), minor allele frequency (MAF $<0.01$ ), and Hardy-Weinberg Equilibrium (HWE p-value less than $\left.1 \times 10^{-6}\right)$. Good quality variants were used as input for imputation to the 1000 Genomes reference panel (phase 1, version 3) using validated software packages (MaCH/minimac, IMPUTE2, BEAGLE, GenABLE). Variants that were poorly imputed $\left(R^{2}<0.5\right)$ or uncommon $(\mathrm{MAF}<0.5 \%)$ were removed before meta-analysis. Full details on the site-specific genotyping and quality control may be found in Supplementary Table 2.

Imaging. Magnetic resonance imaging (MRI) was obtained from scanners with a diversity of manufacturers, field strengths, and acquisition protocols. Images were used to estimate milliliters of intracranial volume from automated segmentations generated by freely available or in-house methods that have been described and validated earlier. Most sites measured intracranial volume for each participant by multiplying the inverse of the determinant of the transformation matrix required to register the subject's MRI scan to a common template by the template volume $\left(1,948,105 \mathrm{~mm}^{3}\right)$, using the FreeSurfer software. Visual inspections were performed to identify and remove poorly segmented images. Either all scans were visually inspected, or sites generated histogram plots to identify any outliers, which were defined as individuals with a volume more than 3 s.d. away from the mean. Statistical outliers were only excluded if the segmentations were deemed improper. More site-specific information related to the imaging is available in Supplementary Table 3.

GWAS. GWAS of intracranial volume were performed for each site separately, controlling for age, sex, and, when applicable, age ${ }^{2}$, population stratification variables (MDS / principal components), study site (for multi-site studies only), diagnosis (for case-control studies only). Studies of unrelated individuals performed a linear regression analyses whereas studies of related individuals (ASPSFam, BrainSCALE, ERF, GeneSTAR, GOBS, NeuroIMAGE, NTR-Adults, OATS, QTIM, SYS) used linear mixed models to account for familial relationships. Summary statistics, including the effect estimates of the genetic variant with intracranial volume under an additive model, were exchanged to perform a fixed-effects meta-analysis weighting for sample size in $\mathrm{METAL}^{47}$. After the final meta-analysis, variants were excluded if they were only available for fewer than 5,000 individuals. Meta-analyses were stratified by race and done separately for discovery, replication, and generalization samples. Beta coefficients were recalculated from Z-scores, allele frequencies, and the sample, as described earlier ${ }^{48}$ Site-specific quantile-quantile plots were generated to inspect the presence of genomic inflation. The variance explained by all variants in the GWAS was estimated using LD score regression ${ }^{12,49}$. Sensitivity analyses were performed by excluding patients.

Functional annotation. All tracks of the regional association plots were taken from the UCSC Genome Browser Human hg19 assembly. SNPs (top 5\%) shows the top $5 \%$ associated variants within the locus and are colored by their correlation to the top variant. Genes shows the gene models from GENCODE version 19. The tracks give the predicted chromatin states based on computational integration of ChIP-seq data for 12 chromatin marks in various human tissues derived from the Roadmap Epigenomics Consortium ${ }^{46}$. In addition, we used HaploReg version 3 for annotation of the top variants and all variants in LD $(>0.80)$ (http://www.broadinstitute.org/mammals/haploreg/haploreg_v3.php).

Genetic correlation. The genetic correlation analyses were also performed using LD score regression. The GWAS meta-analysis of intracranial volume, as well as the height adjusted and height subset meta-analyses, were correlated with published GWAS of the following traits: child head circumference ${ }^{26}$, birth weight ${ }^{50}$, birth length ${ }^{51}$, adult height ${ }^{13}$, childhood cognitive function ${ }^{45}$, adult cognitive function $^{40}$, Alzheimer's disease ${ }^{52}$, Parkinson's disease ${ }^{43}$, white matter lesions ${ }^{29}$, psychiatric disorders $^{44}$, neuroticism ${ }^{41}$, and extraversion ${ }^{42}$.

Enrichment analyses. To determine whether the intracranial volume association results were enriched for certain types of genetic variants, we employed two strategies: partitioned heritability and pathway analyses.

Partitioned heritability was calculated using a previously described method ${ }^{49}$. This was done by partitioning variants by chromosome and by 28 functional classes: coding, UTR, promoter, intron, histone marks H3K4me1, H3K4me3, $\mathrm{H} 3 \mathrm{~K} 9 \mathrm{ac} 5$ and two versions of H3K27ac, open chromatin DNase I hypersensitivity Site (DHS) regions, combined chromHMM/Segway predictions, regions that are conserved in mammals, super-enhancers and active enhancers from the FANTOM5 panel of samples ${ }^{49}$. Multiple testing thresholds were calculated accordingly: $P_{\text {thresh }}=0.05 /(22$ chromosomes $)=2.27 \times 10^{-3}$ for the chromosomes and $P_{\text {thresh }}=0.05 /(28$ classes $)=1.79 \times 10^{-3}$ for the functional classes .

Pathway analyses were performed using the KGG2.5 (ref. 53) and MAGENTA ${ }^{54}$ software packages. LD was calculated based with the 1000 Genomes Project European samples as a reference (see below). Variants were considered to be within a gene if they were within $5 \mathrm{~kb}$ of the $3^{\prime} / 5^{\prime}$ UTR based on chromosome positions (hg19) coordinates. Gene-based tests were done with the GATES test ${ }^{53}$ without weighting $P$ values by predicted functional relevance. Pathway analysis was performed using the HYST test of association ${ }^{55}$. A multiple testing threshold accounting for the number of pathways tested resulting in a significance threshold of $P_{\text {thresh }}=0.05 /(671$ pathways $)=7.45 \times 10^{-5}$.

Head growth trajectories. Head growth trajectory analyses were done within the Generation R study, a longitudinal cohort study situated in Rotterdam, the Netherlands. For this analysis we included 2,824 children of European ancestry followed prenatally until 6 years of age. Head size was measured at the following points: prenatally (using echo) during the first, second, and third trimester, and postnatally (measuring head circumference) at $0-2$ months, 2 months, 3 months, 4 months, 5-10 months, 10-13 months, 13-17 months, and 5 years of age. We tested whether a polygenic score of the 7 loci, as well as the 7 loci themselves separately, were related to head growth using linear mixed models and included an interaction term between time and the genetic score/variant (SAS software). Next, the predicted values were calculated for each person and plotted over time, stratified by genotype ( $0 / 1 / 2$ risk alleles) using the $\mathrm{R}$ software package.

47. Willer, C.J., Li, Y. \& Abecasis, G.R. METAL: fast and efficient meta-analysis of genomewide association scans. Bioinformatics 26, 2190-2191 (2010).

48. Chauhan, G. et al. Association of Alzheimer's disease GWAS loci with MRI markers of brain aging. Neurobiol. Aging 36, 1765.e7-1765.e16 (2015).

49. Finucane, H.K. et al. Partitioning heritability by functional category using GWAS summary statistics. Nat. Genet. 47, 1228-1235 (2015).

50. Horikoshi, M. et al. New loci associated with birth weight identify genetic links between intrauterine growth and adult height and metabolism. Nat. Genet. 45, 76-82 (2013).

51. van der Valk, R.J.P. et al. A novel common variant in DCST2 is associated with length in early life and height in adulthood. Hum. Mol. Genet. 24, 1155-1168 (2015).

52. Lambert, J.-C. et al. Meta-analysis of 74,046 individuals identifies 11 new susceptibility loci for Alzheimer's disease. Nat. Genet. 45, 1452-1458 (2013).

53. Li, M.X., Gui, H.S., Kwan, J.S. \& Sham, P.C. GATES: a rapid and powerful genebased association test using extended Simes procedure. Am. J. Hum. Genet. 88, 283-293 (2011)

54. Segrè, A.V., Groop, L., Mootha, V.K., Daly, M.J. \& Altshuler, D. Common inherited variation in mitochondrial genes is not enriched for associations with type 2 diabetes or related glycemic traits. PLoS Genet. 6, e1001058 (2010).

55. Li, M.X., Kwan, J.S. \& Sham, P.C. HYST: a hybrid set-based test for genome-wide association studies, with application to protein-protein interaction-based association analysis. Am. J. Hum. Genet. 91, 478-488 (2012). 